\title{
Polynomial cubic splines with tension properties
}

\author{
P. Costantini ${ }^{\text {a,*}}$, P.D. Kaklis ${ }^{\text {b }}$, C. Manni ${ }^{\mathrm{c}}$ \\ a Università di Siena, Dipartimento di Scienze Matematiche ed Informatiche, Pian dei Mantellini 44, 53100 Siena, Italy \\ b National Technical University of Athens, Ship-Design Laboratory, Heroon Polytechneiou 9, Zografou 157 73, Athens, Greece \\ c Università di Roma “Tor Vergata”, Dipartimento di Matematica, Via della Ricerca Scientifica, 00133 Roma, Italy
}

\section{A R T I C L E I N F O}

Article history:

Available online 30 June 2010

Keywords:

Polynomial splines

Rational functions

Subdivision schemes

Shape preservation

Tension property

\section{A B S T R A C T}

In this paper we present a new class of spline functions with tension properties. These splines are composed by polynomial cubic pieces and therefore are conformal to the standard, NURBS based CAD/CAM systems.

(c) 2010 Elsevier B.V. All rights reserved.

\section{Introduction}

The popularity and the wide spreading that CAD/CAM systems have nowadays is mostly based on the efficiency of their geometric/mathematic engines. It is well known that the kernel of such systems is constituted by B-splines basis functions (or by their rational counterparts in NURBS) which are used to form curves, tensor product or Boolean sum surfaces. B-splines have several nice properties (positivity, partition of unity, compact support, recurrence relations, etc.) but are not so flexible as required in some applications. If we think, for instance, to interpolation problems, we know that in general spline functions are not shape preserving, that is are not capable of reproducing the shape (sign of discrete curvature and/or torsion) of the data. In the last decades several new classes of piecewise functions of different form and with different properties have been proposed, and it is not possible to mention all of them and all their applications. Nevertheless, a conspicuous part of them have properties similar to standard cubic splines and can be grouped under the name of generalized cubic splines, that is piecewise functions composed by generalized cubic polynomials with the following characteristics. The generalized cubic polynomials are taken from four-dimensional spaces; the generalized cubic polynomials depend on shape parameters, tend to affine functions for their limit values and reduce to standard cubic polynomials for other specific values; generalized splines can be constructed connecting together generalized cubic polynomials. Since the shape parameters can be locally or globally adjusted to give the generalized spline a piecewise linear appearance, they are also refereed to as splines with tension properties. Among the many possible well-known examples we mention here exponential splines (Schweikert, 1966; Späth, 1969; Koch and Lyche, 1993), rational splines (Delbourgo and Gregory, 1985; Delbourgo, 1989), non-uniform degree or variable-degree splines (Kaklis and Pandelis, 1990; Kaklis and Sapidis, 1995; Costantini, 2000).

\footnotetext{
Work supported by INdAM - Gruppo Nazionale Calcolo Scientifico.

* Corresponding author.

E-mail addresses: costantini@unisi.it (P. Costantini), kaklis@deslab.ntua.gr (P.D. Kaklis), manni@mat.uniroma2.it (C. Manni).
} 
Unfortunately, commercial CAD/CAM systems are very conservative, in the sense that they are constructed to support only low degree polynomial splines and NURBS. In particular, they cannot accept the above mentioned generalized splines, which are either not polynomial or polynomial with arbitrary degree. As a serious consequence, CAD/CAM systems renounce to incorporate the recent scientific acquisitions and to gain a stronger control on the shape of curves or surfaces.

The goal of the present paper is to give a first answer in this direction, describing a new set of generalized splines with tension properties which are composed by $C^{2}$ cubic polynomial pieces and thus can be perfectly integrated in every CAD/CAM system. The tools adopted are strictly connected to the Hermite subdivision scheme described in Costantini and Manni (2010b) and based in turn on the more general results of Costantini and Manni (2010a). More specifically, in Costantini and Manni (2010b) it is shown that it is possible to construct a space of $C^{2}$ generalized splines, equipped with tension properties, chiefly composed by cubic pieces with the exception of some special rational pieces. Here we show how to modify the rational pieces in cubic polynomial ones while maintaining all the desired properties.

This paper is divided in five sections. In the next one we recall from Costantini et al. (2005), Costantini and Manni (2006), the basic information on generalized cubic polynomials and generalized splines, adding some new material on splines with multiple knots. The third section describes the main results of the paper, that is the substitution of the rational pieces with cubic polynomials. Section 4 describes the spline space and the construction of the B-spline basis with tension properties. Section 5 is devoted to show some examples of applications (limited, for reasons of space, to curve and surface design), and to final comments and remarks.

\section{Some preliminary material}

In this section we collect the material necessary for the comprehension of the paper.

\subsection{Generalized cubics}

Given a real function $f$, we denote with $\dot{f}$ the derivative w.r. to the (local) variable $t$ and with $f^{\prime}$ or $D f$ the derivative w.r. to the (global) variable $x$. Assume, for notational simplicity, $t \in[0,1]$ and let

$$
\mathcal{P}_{u, v}:=\langle 1, t, u(t), v(t)\rangle, \quad u, v \in C^{2}[0,1], \quad \operatorname{dim}\left(\mathcal{P}_{u, v}\right)=4
$$

We state the following ${ }^{1}$

Definition 1. A basis $\left\{B_{0}, B_{1}, B_{2}, B_{3}\right\}$ of $\mathcal{P}_{u, v}$ is a Bernstein like basis if it gives a partition of unity, is totally positive ${ }^{2}$ on $[0,1]$ and it satisfies

$$
\begin{array}{ll}
B_{0}(1)=\dot{B}_{0}(1)=\ddot{B}_{0}(1)=0, & B_{1}(0)=B_{1}(1)=\dot{B}_{1}(1)=0, \\
B_{2}(0)=\dot{B}_{2}(0)=B_{2}(1)=0, & B_{3}(0)=\dot{B}_{3}(0)=\ddot{B}_{3}(0)=0 .
\end{array}
$$

Assuming that $\mathcal{P}_{u, v}$ has a Bernstein like basis, we denote by $\xi_{0}, \xi_{1}, \xi_{2}, \xi_{3}$ the Greville abscissas, that is $t=\sum_{q=0}^{3} \xi_{q} B_{q}(t)$. In the following we assume

$$
0=\xi_{0}<\xi_{1}<\xi_{2}<\xi_{3}=1
$$

and we associate to any element $\psi$ of $\mathcal{P}_{u, v}, \psi(t):=\sum_{q=0}^{3} b_{q} B_{q}(t)$, its Bézier control polygon (or simply control polygon) that is the polygonal line $\ell$ with vertices (Bézier control points) $\left(\xi_{0}, b_{0}\right),\left(\xi_{1}, b_{1}\right),\left(\xi_{2}, b_{2}\right),\left(\xi_{3}, b_{3}\right)$. The Bézier control polygon closely imitates the shape of $\psi$, in the sense specified in Peña (1995), Chapter 3.

From the more general results of Costantini et al. (2005), Goodman and Mazure (2001), Costantini and Manni (2006), we infer the following proposition.

Proposition 1. If the functions $u$ and $v$ are such that

$\{\ddot{u}, \ddot{v}\} \quad$ is a Chebyshev System in $[0,1]$,

then $\mathcal{P}_{u, v}$ has a Bernstein like basis and (3) holds.

Without loss of generality, we can put $u=B_{0}, v=B_{3}$. If (4) holds, $\mathcal{P}_{u, v}$ "behaves" as cubic polynomials ( $\left.\mathbb{P}_{3}\right)$ and reduces to it when $u(t):=(1-t)^{3}, v(t):=t^{3}$. For this reason spaces $\mathcal{P}_{u, v}$ are usually referred to as generalized cubics.

\footnotetext{
${ }^{1}$ In this context we are dealing with functions of class $C^{2}$. In case spaces of less regularity are of interest, different definitions of Bernstein like bases can be provided. For $H C^{1}$ subdivision schemes see, e.g., Sablonnière (2004).

${ }^{2}$ A sequence of functions $\left\{\phi_{0}, \ldots, \phi_{n}\right\}$ is totally positive on a given interval $I$ if for any points $t_{0}<t_{1}<\cdots<t_{m}$ in $I$ the collocation matrix $\left(\phi_{j}\left(t_{i}\right)\right)_{i=0 j=0}^{m}$ is totally positive, i.e. all its minors are nonnegative.
} 
We recall from Costantini and Manni (2010b) an alternative expression for $\psi$ useful in the sequel:

$$
\psi(x)=e_{0} B_{0}(x)+r(x)+e_{1} B_{3}(x)=e_{0} u(x)+r(x)+e_{1} v(x),
$$

where

$$
r(x):=\frac{b_{2}-b_{1}}{\xi_{2}-\xi_{1}}\left(x-\xi_{1}\right)+b_{1}, \quad e_{0}:=b_{0}-r(0), \quad e_{1}:=b_{3}-r(1)
$$

and the following properties

$$
\begin{array}{lll}
\psi\left(\xi_{0}\right)=b_{0}, & \dot{\psi}\left(\xi_{0}\right)=\frac{\left(b_{1}-b_{0}\right)}{\left(\xi_{1}-\xi_{0}\right)}, & \ddot{\psi}\left(\xi_{0}\right)=e_{0} \ddot{u}(0), \\
\psi\left(\xi_{3}\right)=b_{3}, & \dot{\psi}\left(\xi_{3}\right)=\frac{\left(b_{3}-b_{2}\right)}{\left(\xi_{3}-\xi_{2}\right)}, & \ddot{\psi}\left(\xi_{3}\right)=e_{1} \ddot{v}(1) .
\end{array}
$$

In many practical applications, for the definition of (1) functions of the form

$$
u=u(t ; \alpha), \quad v=v(t ; \beta)
$$

are used, where $\alpha, \beta$ are tension parameters, ${ }^{3}$ that is are such that

$$
\begin{aligned}
& u(t ; 3)=(1-t)^{3}, \quad v(t ; 3)=t^{3}, \\
& \lim _{\alpha \rightarrow+\infty} u(t)=\lim _{\alpha \rightarrow+\infty} \dot{u}(t)=\lim _{\alpha \rightarrow+\infty} \ddot{u}(t)=0, \quad t \in[a, 1], a>0, \\
& \lim _{\beta \rightarrow+\infty} v(t)=\lim _{\beta \rightarrow+\infty} \dot{v}(t)=\lim _{\beta \rightarrow+\infty} \ddot{v}(t)=0, \quad t \in[0, b], b<1 .
\end{aligned}
$$

Moreover,

$$
\alpha=-\dot{u}(0)=-\dot{B}_{0}(0), \quad \beta=\dot{v}(1)=\dot{B}_{3}(1),
$$

so that, because of (2),

$$
\xi_{1}=\xi_{0}+\frac{1}{\alpha}, \quad \xi_{2}=\xi_{3}-\frac{1}{\beta} .
$$

In particular, assume $\psi$ is the solution of the Hermite problem

$$
\psi(0)=f_{0}, \quad \dot{\psi}(0)=d_{0}, \quad \psi(1)=f_{1}, \quad \dot{\psi}(1)=d_{1},
$$

then

$$
b_{0}=f_{0}, \quad b_{1}=f_{0}+\frac{d_{0}}{\alpha}, \quad b_{2}=f_{1}-\frac{d_{1}}{\beta}, \quad b_{3}=f_{1},
$$

and both the control polygon and the function $\psi$ tend to the straight line interpolating the points $\left(0, f_{0}\right),\left(1, f_{1}\right)$. If we have the data in non-decreasing and/or non-convex position

$$
f_{0} \leqslant f_{1}, \quad d_{0} \geqslant 0, \quad d_{1} \geqslant 0 \text { and/or } \quad d_{0} \leqslant f_{1}-f_{0} \leqslant d_{1},
$$

it suffices to increase the values of $\alpha, \beta$ in order to push the control points in non-decreasing and/or non-convex position. Since the generalized cubics reproduce the shape of their control polygons, it follows that $\psi$ has the same shape of the data.

\subsection{An example: rational functions}

Let us consider the space of rational functions

$$
R^{\mu, v}=\langle 1, t, u(t ; \mu), v(t ; v)\rangle, \quad \mu, v \geqslant 3
$$

where $t \in[0,1]$ and

\footnotetext{
3 There are famous classes of functions which assume the limit form for different values of the tension parameters and have more complicate expressions for the derivatives at end points. For instance, exponential functions (Schweikert, 1966) approach the cubic ones when both $\alpha$ and $\beta$ tend to zero and the derivatives are transcendental functions in $\alpha$ and $\beta$.
} 


$$
u(t)=u(t ; \mu):=\frac{(1-t)^{3}}{1+(\mu-3) t(1-t)}, \quad v(t)=v(t ; v):=\frac{t^{3}}{1+(v-3)(1-t) t}
$$

It is well known that $R^{\mu, v}$ is a four-dimensional space, that $R^{3,3} \equiv \mathbb{P}_{3}$ and that it is possible to find a unique $\psi \in R^{\mu, \nu}$ which satisfies the Hermite interpolation problem (7). Spaces of the form (8) are fundamental in the following section and have been introduced several years ago (see, e.g., the pioneering papers Delbourgo and Gregory, 1985; Delbourgo, 1989) to solve shape-preserving interpolation problems. $\mu$ and $v$ act as tension parameters; indeed, if $\psi$ satisfies (7) and $\rho$ is the straight line trough the points $\left(0, f_{0}\right),\left(1, f_{1}\right)$ then

$$
\lim _{\mu, \nu \rightarrow \infty}\|\psi-\rho\|_{\infty}=0 .
$$

Functions of the form (9) satisfy (4); therefore the space (8) admits a Bernstein basis and can be seen as a generalized cubic space.

\subsection{Generalized splines}

In this subsection we describe the construction of cubic-like B-spline bases obtained connecting functions taken from spaces of the form (1). We start recalling from Costantini and Manni (2006) the construction of splines with simple knots and then we will give the main ideas for multiple knots.

Let us consider the (extended) sequence of knots

$$
\bar{x}_{-3}=\cdots=\bar{x}_{0} \leqslant \cdots \leqslant \bar{x}_{N}=\cdots=\bar{x}_{N+3},
$$

let

$$
\bar{y}_{0}<\bar{y}_{1}<\cdots<\bar{y}_{n}
$$

be the subset of distinct knots, with multiplicity $m_{0}, \ldots, m_{n}$, where $\sum_{i=0}^{n} m_{i}=N+7$. For any $i=0, \ldots, n-1$ let us consider the corresponding grid-spacings $\bar{h}_{i}:=\bar{y}_{i+1}-\bar{y}_{i}$, a pair of functions

$$
u_{i}=u_{i}(t), \quad v_{i}=v_{i}(t), \quad t=\left(x-\bar{y}_{i}\right) / \bar{h}_{i}
$$

and the corresponding spaces $\mathcal{P}_{u_{i}, v_{i}}$ (see (1)). Let $\mathcal{U}$ and $\mathcal{V}$ denote, respectively, the set of functions $u_{i}$ and $v_{i}$. Finally, let us consider the space of functions defined in $\left[\bar{y}_{0}, \bar{y}_{n}\right]$ belonging "piecewise" to $\mathcal{P}_{u_{i}, v_{i}}$, that is

$$
S_{\mathcal{U}, \mathcal{V}}:=\left\{s \text { s.t. } s_{i}:=s_{\mid\left[\bar{y}_{i}, \bar{y}_{i+1}\right)} \in \mathcal{P}_{u_{i}, v_{i}}, i=0, \ldots, n-1 ; s \in C^{3-m_{i}}\left(\bar{y}_{i}\right), i=1, \ldots, n-1\right\} .
$$

Let us assume that $\mathcal{P}_{u_{i}, v_{i}}$ possesses a Bernstein like basis (see Definition 1) $\left\{B_{i, 0}, B_{i, 1}, B_{i, 2}, B_{i, 3}\right\}$ satisfying (3). For each segment $\left[\bar{y}_{i}, \bar{y}_{i+1}\right]$ we have $s(x)=s_{i}(t)=\sum_{q=0}^{3} b_{i, q} B_{i, q}(t)$ and the abscissas of control points are denoted with $\xi_{i, q}, q=$ $0,1,2,3$; moreover, we assume $B_{i, 0}=u_{i}, B_{i, 3}=v_{i}$. The abscissas of the $m_{i}$ de Boor control points associated with the knot $\bar{y}_{i}$ are denoted with $\sigma_{i}^{\zeta_{i}}, \zeta_{i}=1, \ldots, m_{i}$. For a given index $i$, assume, for the moment, $m_{i-2}=\cdots=m_{i+2}=1$. Clearly $s$ is continuous in $\bar{y}_{i}$ if and only if

$$
b_{i-1,3}=b_{i, 0} .
$$

Setting

$$
\rho_{i}:=-\frac{1}{\dot{u}_{i}(0)}=\frac{1}{\alpha_{i}}, \quad \omega_{i}:=\frac{1}{\dot{v}_{i}(1)}=\frac{1}{\beta_{i}}, \quad \gamma_{i}:=1-\rho_{i}-\omega_{i},
$$

we have that $s^{\prime}\left(\bar{y}_{i}^{-}\right)=s^{\prime}\left(\bar{y}_{i}^{+}\right)$if and only if

$$
\left(b_{i-1,3}-b_{i-1,2}\right) \frac{1}{\bar{h}_{i-1} \omega_{i-1}}=\left(b_{i, 1}-b_{i, 0}\right) \frac{1}{\bar{h}_{i} \rho_{i}},
$$

while $s^{\prime \prime}\left(\bar{y}_{i}^{-}\right)=s^{\prime \prime}\left(\bar{y}_{i}^{+}\right)$if and only if

$$
\frac{\ddot{v}_{i-1}(1)}{\bar{h}_{i-1}^{2}}\left[\left(b_{i-1,3}-b_{i-1,2}\right)-\left(b_{i-1,2}-b_{i-1,1}\right) \frac{\omega_{i-1}}{\gamma_{i-1}}\right]=\frac{\ddot{u}_{i}(0)}{\bar{h}_{i}^{2}}\left[\left(b_{i, 2}-b_{i, 1}\right) \frac{\rho_{i}}{\gamma_{i}}-\left(b_{i, 1}-b_{i, 0}\right)\right] .
$$

Finally, we set

$$
\begin{aligned}
\vartheta_{i}^{+} & :=\frac{\bar{h}_{i-1}}{\bar{h}_{i}} \frac{\rho_{i}}{\gamma_{i}} \ddot{u}_{i}(0) \vartheta_{i}, \quad \vartheta_{i}^{-}:=\frac{\bar{h}_{i}}{\bar{h}_{i-1}} \frac{\omega_{i-1}}{\gamma_{i-1}} \ddot{v}_{i-1}(1) \vartheta_{i}, \quad \vartheta_{i}:=\frac{\bar{h}_{i-1} \omega_{i-1}+\bar{h}_{i} \rho_{i}}{\ddot{v}_{i-1}(1) \bar{h}_{i} \omega_{i-1}+\ddot{u}_{i}(0) \bar{h}_{i-1} \rho_{i}}, \\
\sigma_{i}^{1} & :=\bar{x}_{i}+\rho_{i}-\vartheta_{i}^{+} \bar{h}_{i} \gamma_{i}=\bar{x}_{i}-\omega_{i-1} \bar{h}_{i-1}+\vartheta_{i}^{-} \gamma_{i-1} \bar{h}_{i-1} .
\end{aligned}
$$



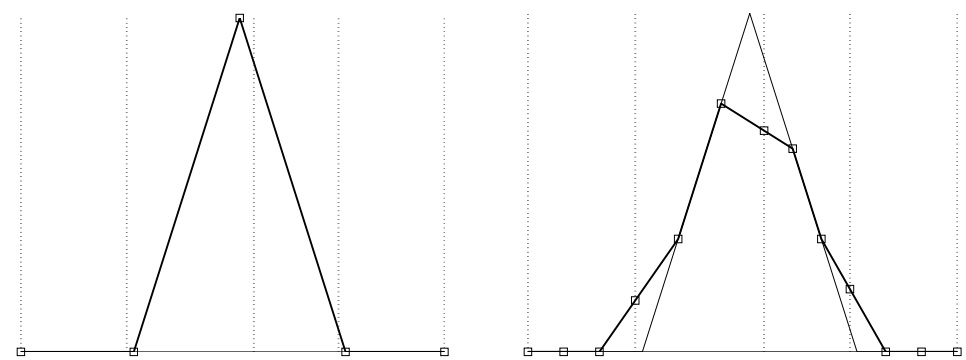

Fig. 1. The de Boor (left) and the Bézier (right) control polygons for the B-spline with $C^{2}$ continuity associated with the knot $\bar{y}_{i}$.
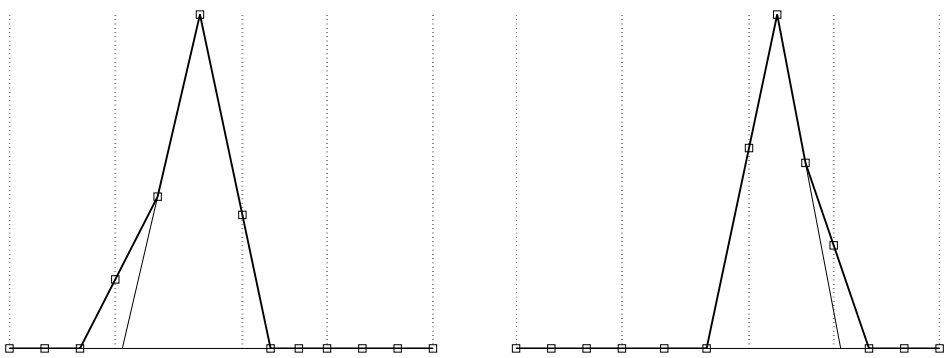

Fig. 2. The de Boor and Bézier control polygons for the two B-splines with $C^{1}$ continuity associated with the knot $\bar{y}_{i}$.

Repeating this arguments for the indices $i-2, \ldots, i+2$, we can associate to the knot $\bar{y}_{i}$ a polygonal line (the generalized de Boor control polygon) which vanishes for $x<\sigma_{i-2}^{1}, x>\sigma_{i+2}^{1}$, has vertices

$$
\left(\sigma_{\tau}^{1}, P_{\tau}^{1}\right), \quad \tau=i-2, \ldots, i+2 ; \quad P_{\tau}^{1}= \begin{cases}1 & \text { for } \tau=i, \\ 0 & \text { for } \tau=i-2, i-1, i+1, i+2,\end{cases}
$$

so that the Bézier coefficients of $s$ can be deduced according to the following rules

$$
\begin{aligned}
& b_{\tau-1,2}=P_{\tau}^{1} \frac{1+\vartheta_{\tau-1}^{+}}{1+\vartheta_{\tau-1}^{+}+\vartheta_{\tau}^{-}}+P_{\tau-1}^{1} \frac{\vartheta_{\tau}^{-}}{1+\vartheta_{\tau-1}^{+}+\vartheta_{\tau}^{-}}, \\
& b_{\tau, 1}=P_{\tau}^{1} \frac{1+\vartheta_{\tau+1}^{-}}{1+\vartheta_{\tau+1}^{-}+\vartheta_{\tau}^{+}}+P_{\tau+1}^{1} \frac{\vartheta_{\tau}^{+}}{1+\vartheta_{\tau+1}^{-}+\vartheta_{\tau}^{+}}, \\
& b_{\tau-1,3}=b_{\tau, 0}=b_{\tau-1,2} \frac{\bar{h}_{\tau} \rho_{\tau}}{\rho_{\tau} \bar{h}_{\tau}+\omega_{\tau-1} \bar{h}_{\tau-1}}+b_{\tau, 1} \frac{\bar{h}_{\tau-1} \omega_{\tau-1}}{\rho_{\tau} \bar{h}_{\tau}+\omega_{\tau-1} \bar{h}_{\tau-1}},
\end{aligned}
$$

for $\tau=i-1, i, i+1$ and $b_{\tau, q}=0$ elsewhere. The above formulas are completely similar to the classical geometric construction for $C^{2}$ cubic splines (see, e.g., Hoschek and Lasser, 1993) and, if applied to the control points (16) give rise to the Bézier control points of an element of the B-spline basis. Such construction is explained in Fig. 1.

If we assume $m_{i}=2$ then the only (13) and (14) must be satisfied. We can associate two de Boor control polygons with the knot $\bar{y}_{i}$ as shown in Fig. 2 . The abscissas of the de Boor control points are given by

$$
\sigma_{i}^{1}=\xi_{i-1,2}=\bar{y}_{i}-\bar{h}_{i-1} \omega_{i-1}, \quad \sigma_{i}^{2}=\xi_{i, 1}=\bar{y}_{i}+\bar{h}_{i} \rho_{i} .
$$

The non-zero control points corresponding to the figure on the left are given by

$$
b_{i-1,2}=1, \quad b_{i-1,1}=\frac{1}{\sigma_{i}^{1}-\sigma_{i-1}^{1}}\left(\xi_{i-1,1}-\sigma_{i-1}^{1}\right),
$$

and $b_{i-2,3}=b_{i-1,0}$ and $b_{i-1,3}=b_{i, 0}$ can be computed as in (17). Note that $b_{i-1,2}$ gives the de Boor ordinate associated with $\sigma_{i}^{1}$. The construction for the control points of the figure on the right is symmetric.

If we assume $m_{i}=3$ then we must satisfy only (13). The abscissas of the de Boor control points are given by

$$
\sigma_{i}^{1}=\xi_{i-1,2}=\bar{y}_{i}-\bar{h}_{i-1} \omega_{i-1}, \quad \sigma_{i}^{2}=\xi_{i-1,3}=\xi_{i, 0}=\bar{y}_{i}, \quad \sigma_{i}^{3}=\xi_{i, 1}=\bar{y}_{i}+\bar{h}_{i} \rho_{i} .
$$

The three functions associated to $\bar{y}_{i}$ are plotted in Fig. 3. The non-zero control points for the first and last figures, namely $b_{i-2,3}=b_{i-1,0}, b_{i-1,1}, b_{i-1,2}$ for the left and $b_{i, 1}, b_{i, 2}, b_{i, 3}=b_{i+1,0}$ for the right are computed as in the $C^{1}$ case. The only 

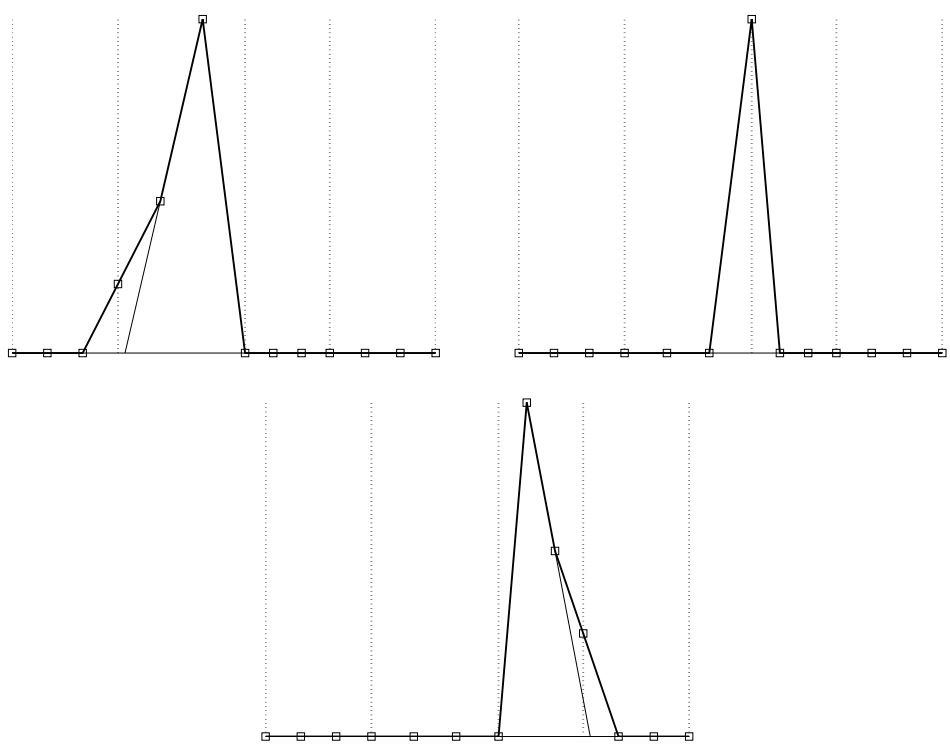

Fig. 3. The de Boor and the Bézier control polygons for the three B-splines with $C^{0}$ continuity associated with the knot $\bar{y}_{i}$.
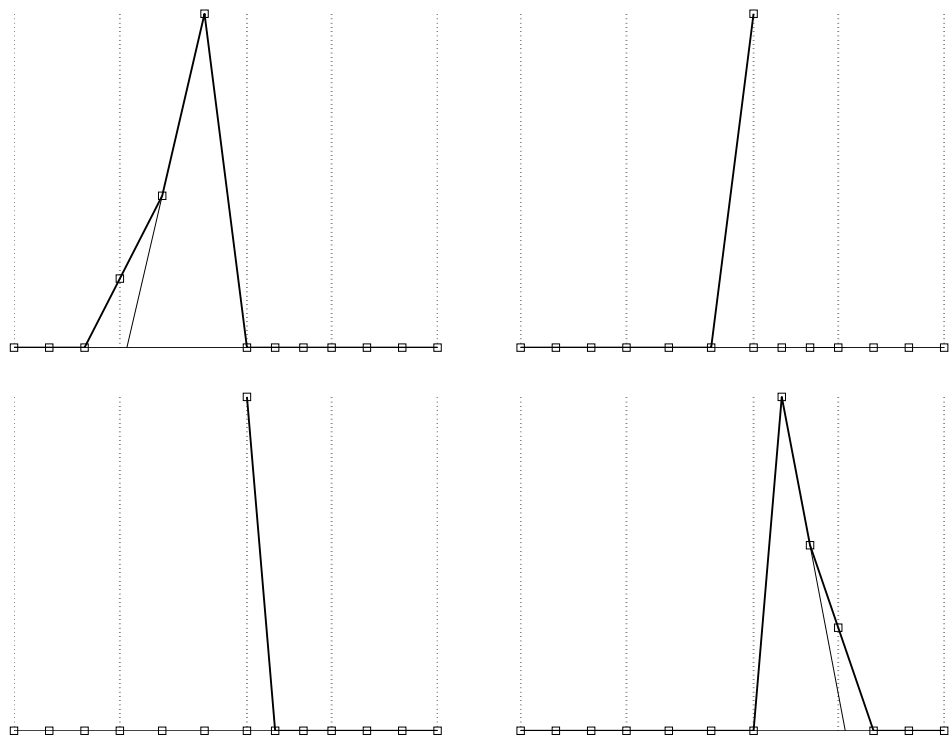

Fig. 4. The Bézier control polygons for the four B-splines with $C^{-1}$ continuity associated with the knot $\bar{y}_{i}$.

non-zero control point of the mid one is $b_{i-1,3}=b_{i, 0}=1$. For the sake of completeness, in Fig. 4 are reported the four control polygon in the case $m_{i}=4$.

The other cases, where $m_{i-2}, m_{i-1}, m_{i+1}, m_{i+2} \neq 1$, can be easily obtained with similar constructions.

In conclusion, it is possible to define a family of functions $\left\{\mathcal{N}_{i}, i=-1, \ldots, N+1\right\}$, which form a basis for the space $S_{\mathcal{U}, \mathcal{V}}$, with the usual properties of the family of cubic B-splines. They will be referred to as generalized cubic B-splines. In particular we have

$$
\mathcal{N}_{i}(x) \geqslant 0, \quad x \in\left[\bar{x}_{-3}, \bar{x}_{N+3}\right] ; \quad \sum_{i=-1}^{N+1} \mathcal{N}_{i}(x) \equiv 1, \quad x \in\left[\bar{x}_{0}, \bar{x}_{N}\right]
$$

\section{Two new spaces of generalized cubics}

In this section we will introduce two new spaces of generalized cubics, $E \mathcal{R}^{(j)}$, composed by $C^{2}$ rational pieces, and $E \mathcal{C}^{(j)}$, composed by $C^{2}$ cubic polynomial pieces. 


\subsection{The space of rational functions $E \mathcal{R}^{(j)}$}

Let us define $\mathcal{D}^{(0)}:=\{0,1\}$; the dyadic points at level $j$ will be denoted with

$$
\mathcal{D}^{(j)}:=\left\{x_{k}^{(j)}:=k h_{j}, k=0, \ldots, 2^{j}\right\}, \quad h_{j}:=2^{-j} .
$$

We take the starting values $\mu_{0}^{(0)}, v_{0}^{(0)} \in \mathbb{R} ; \mu_{0}^{(0)}, v_{0}^{(0)} \geqslant 3$ and generate the sequences of parameters $\mu_{k}^{(j)}, v_{k}^{(j)}, k=0, \ldots$, $2^{j}-1, j=1,2, \ldots$, by the rule

$$
\begin{aligned}
& \left(\mu_{0}^{(j)}, v_{0}^{(j)}\right):=\left(\frac{\mu_{0}^{(j-1)}+3}{2}, 3\right), \\
& \left(\mu_{k}^{(j)}, v_{k}^{(j)}\right):=(3,3), \quad k=1, \ldots, 2^{j}-2, \\
& \left(\mu_{2^{j}-1}^{(j)}, v_{2^{j}-1}^{(j)}\right):=\left(3, \frac{v_{2^{j-1}-1}^{(j-1)}+3}{2}\right) .
\end{aligned}
$$

For each subinterval $\left[x_{k}^{(j)}, x_{k+1}^{(j)}\right]$ at a given level $j$ we consider spaces of the form (8)

$$
R_{k}^{(j)}:=R^{\mu, v}, \quad \mu=\mu_{k}^{(j)}, \quad v=v_{k}^{(j)} ; \quad t=\frac{x-x_{k}^{(j)}}{h_{j}},
$$

and construct a sequence of functions

$$
\psi_{k}^{(j)}, \quad k=0, \ldots, 2^{j}-1, j=0,1, \ldots ; \quad \psi_{k}^{(j)} \in R_{k}^{(j)},
$$

defined recursively as the solutions of the following Hermite interpolation problems. Let $m_{k}^{(j)}=x_{2 k+1}^{(j+1)}:=\left(x_{k}^{(j)}+x_{k+1}^{(j)}\right) / 2$; then

$$
\begin{array}{ll}
\psi_{2 k}^{(j+1)}\left(x_{k}^{(j)}\right)=\psi_{k}^{(j)}\left(x_{k}^{(j)}\right), & D \psi_{2 k}^{(j+1)}\left(x_{k}^{(j)}\right)=D \psi_{k}^{(j)}\left(x_{k}^{(j)}\right), \\
\psi_{2 k}^{(j+1)}\left(m_{k}^{(j)}\right)=\psi_{k}^{(j)}\left(m_{k}^{(j)}\right), & D \psi_{2 k}^{(j+1)}\left(m_{k}^{(j)}\right)=D \psi_{k}^{(j)}\left(m_{k}^{(j)}\right), \\
\psi_{2 k+1}^{(j+1)}\left(m_{k}^{(j)}\right)=\psi_{k}^{(j)}\left(m_{k}^{(j)}\right), & D \psi_{2 k+1}^{(j+1)}\left(m_{k}^{(j)}\right)=D \psi_{k}^{(j)}\left(m_{k}^{(j)}\right), \\
\psi_{2 k+1}^{(j+1)}\left(x_{k+1}^{(j)}\right)=\psi_{k}^{(j)}\left(x_{k+1}^{(j)}\right), & D \psi_{2 k+1}^{(j+1)}\left(x_{k+1}^{(j)}\right)=D \psi_{k}^{(j)}\left(x_{k+1}^{(j)}\right) .
\end{array}
$$

Formulas (22) say that $\psi_{k}^{(j)}$, the generic function at level $j$, is replaced by two new ones, $\psi_{2 k}^{(j+1)}, \psi_{2 k+1}^{(j+1)}$, at level $j+1$ which are the Hermite interpolating functions respectively in the first and second half of the interval $\left[x_{k}^{(j)}, x_{k+1}^{(j)}\right]$. This subdivision process, which is called Hermite $C^{1}$ subdivision has been firstly introduced by Merrien (Merrien, 1992) and then studied by several authors. The interested reader is referred to the references reported in Costantini and Manni (2010a). Since at any stage we use rational functions, the process described by (21) and (22) will be referred to as rational Hermite $C^{1}$ scheme. Note, in particular, that if

$$
\mu_{k}^{(j)}=\mu_{2 k}^{(j+1)}=\mu_{2 k+1}^{(j+1)}=3, \quad v_{k}^{(j)}=v_{2 k}^{(j+1)}=v_{2 k+1}^{(j+1)}=3,
$$

then

$$
\psi_{k}^{(j)}, \psi_{2 k}^{(j+1)}, \psi_{2 k+1}^{(j+1)} \in \mathbb{P}_{3},
$$

that is the function $\psi_{k}^{(j)}$ does not change at the step $j+1$. For any $j$ let $\Psi^{(j)}:[0,1] \mapsto \mathbb{R}$ be the piecewise function defined by

$$
\Psi^{(j)}(x):=\psi_{k}^{(j)}(x), \quad x \in\left[x_{k}^{(j)}, x_{k+1}^{(j)}\right] ;
$$

from (19) and from the above considerations it follows that

$$
\Psi^{(j)}(x)=\Psi^{(j+1)}(x), \quad x \in\left[x_{1}^{(j)}, x_{2^{j-1}}^{(j)}\right],
$$

and therefore

$$
\begin{aligned}
& \Psi^{(2)} \in \mathbb{P}_{3}, \quad x \in I^{(2)}:=\left(x_{1}^{(2)}, x_{2}^{(2)}\right) \cup\left(x_{2}^{(2)}, x_{3}^{(2)}\right), \\
& \Psi^{(3)} \in \mathbb{P}_{3}, \quad x \in I^{(3)}:=\left(x_{1}^{(3)}, x_{1}^{(2)}\right) \cup I^{(2)} \cup\left(x_{3}^{(2)}, x_{7}^{(3)}\right),
\end{aligned}
$$


and, at a general level $j+1$

$$
\Psi^{(j+1)} \in \mathbb{P}_{3}, \quad x \in I^{(j+1)}:=\left(x_{1}^{(j+1)}, x_{1}^{(j)}\right) \cup I^{(j)} \cup\left(x_{2^{j-1}}^{(j)}, x_{2^{j+1}-1}^{(j+1)}\right) .
$$

In Costantini and Manni (2010a) it is proven that the pieces $\psi_{k}^{(j)}$ connect with $C^{2}$ continuity at the internal points of the set (18), or in other words

$$
\Psi^{(j)} \in C^{2}\left[x_{0}^{(j)}, x_{2^{j}}^{(j)}\right] \equiv C^{2}[0,1]
$$

Since in each subinterval $\left[x_{k}^{(j)}, x_{k+1}^{(j)}\right]$ we consider functions of the form (8), (20) for which a Bernstein-Bézier representation exists, the process given by (19)-(22) can be described also in terms of subdivision of the initial control polygon. Keeping in mind Section 2.1, let

$$
\left(\xi_{k, q}^{(j)}, b_{k, q}^{(j)}\right), \quad q=0,1,2,3
$$

be the control points of $\psi_{k}^{(j)}$. Let $\ell_{k}^{(j)}$ such that

$$
\ell_{k}^{(j)}\left(\xi_{k, q}^{(j)}\right)=b_{k, q}^{(j)}, \quad q=0,1,2,3
$$

be the control polygon of $\psi_{k}^{(j)}$ and let $\mathcal{L}_{R}^{(j)}:[0,1] \mapsto \mathbb{R}$ be such that

$$
\mathcal{L}_{R}^{(j)}(x):=\ell_{k}^{(j)}(x), \quad x \in\left[x_{k}^{(j)}, x_{k+1}^{(j)}\right],
$$

the composite control polygon of $\Psi^{(j)}$. Moreover, let

$$
\xi^{(j)}=\left[\xi_{0,0}^{(j)}, \ldots, \xi_{0,3}^{(j)}, \xi_{1,0}^{(j)}, \ldots, \xi_{2^{j}-2,3}^{(j)}, \xi_{2^{j}-1,0}^{(j)}, \ldots, \xi_{2^{j}-1,3}^{(j)}\right]^{T}
$$

and

$$
\boldsymbol{b}^{(j)}=\left[b_{0,0}^{(j)}, \ldots, b_{0,3}^{(j)}, b_{1,0}^{(j)}, \ldots, b_{2^{j}-2,3}^{(j)}, b_{2^{j}-1,0}^{(j)}, \ldots, b_{2^{j}-1,3}^{(j)}\right]^{T},
$$

the sequence of the composite control points of $\Psi^{(j)}$, where $\xi_{k, 3}^{(j)}=\xi_{k+1,0}^{(j)}, b_{k, 3}^{(j)}=b_{k+1,0}^{(j)}$. For notational convenience we introduce the linear operator $\mathcal{B}_{R}^{(j)}$ such that

$$
\Psi^{(j)}=\mathcal{B}_{R}^{(j)} \boldsymbol{b}^{(j)}
$$

In Costantini and Manni (2010a, 2010b) it is proven that

$$
\begin{aligned}
& {\left[\begin{array}{llllllll}
\xi_{2 k, 0}^{(j+1)} & \xi_{2 k, 1}^{(j+1)} & \xi_{2 k, 2}^{(j+1)} & \xi_{2 k, 3}^{(j+1)} & \xi_{2 k+1,0}^{(j+1)} & \xi_{2 k+1,1}^{(j+1)} & \xi_{2 k+1,2}^{(j+1)} & \xi_{2 k+1,3}^{(j+1)} \\
b_{2 k, 0}^{(j+1)} & b_{2 k, 1}^{(j+1)} & b_{2 k, 2}^{(j+1)} & b_{2 k, 3}^{(j+1)} & b_{2 k+1,0}^{(j+1)} & b_{2 k+1,1}^{(j+1)} & b_{2 k+1,2}^{(j+1)} & b_{2 k+1,3}^{(j+1)}
\end{array}\right]^{T}} \\
& =S_{k}^{(j)}\left[\begin{array}{cccc}
\xi_{k, 0}^{(j)} & \xi_{k, 1}^{(j)} & \xi_{k, 2}^{(j)} & \xi_{k, 3}^{(j)} \\
b_{k, 0}^{(j)} & b_{k, 1}^{(j)} & b_{k, 2}^{(j)} & b_{k, 3}^{(j)}
\end{array}\right]^{T},
\end{aligned}
$$

with

$$
S_{k}^{(j)}:=S_{k, 4}^{(j)} S_{k, 3}^{(j)} S_{k, 2}^{(j)} S_{k, 1}^{(j)}, \quad S_{k}^{(j)} \in \mathbb{R}^{8 \times 4},
$$

where $S_{k, 1}^{(j)} \in \mathbb{R}^{5 \times 4}, S_{k, 2}^{(j)} \in \mathbb{R}^{6 \times 5}, S_{k, 3}^{(j)} \in \mathbb{R}^{7 \times 6}$ are given in Costantini and Manni (2010b), p. 1663, and

$$
S_{k, 4}^{(j)}:=\left[\begin{array}{lllllll}
1 & 0 & 0 & 0 & 0 & 0 & 0 \\
0 & 1 & 0 & 0 & 0 & 0 & 0 \\
0 & 0 & 1 & 0 & 0 & 0 & 0 \\
0 & 0 & 0 & 1 & 0 & 0 & 0 \\
0 & 0 & 0 & 1 & 0 & 0 & 0 \\
0 & 0 & 0 & 0 & 1 & 0 & 0 \\
0 & 0 & 0 & 0 & 0 & 1 & 0 \\
0 & 0 & 0 & 0 & 0 & 0 & 1
\end{array}\right] .
$$

Summarizing, we consider the subdivision scheme defined as

$$
\left[\xi^{(j)}, \boldsymbol{b}^{(j)}\right]:=S^{(j)} S^{(j-1)} \ldots S^{(1)} S^{(0)}\left[\xi^{(0)}, \boldsymbol{b}^{(0)}\right]
$$


where each $S^{(p)}, p=0, \ldots, j$, has the form

$$
S^{(p)}=\left[\begin{array}{ccccc}
S_{0}^{(p)} & 0 & \cdots & 0 & 0 \\
0 & S_{1}^{(p)} & \cdots & 0 & 0 \\
\vdots & \vdots & \ddots & \vdots & \vdots \\
0 & 0 & \cdots & S_{2^{p}-2}^{(p)} & 0 \\
0 & 0 & \cdots & 0 & S_{2^{p}-1}^{(p)}
\end{array}\right], \quad S^{(p)} \in \mathbb{R}^{82^{p} \times 42^{p}} .
$$

The following results were proven in Costantini and Manni (2010a).

Proposition 2. The elements of the sequences defined by (27), (28) are such that

$$
\xi_{k, 0}^{(j)}=x_{k}^{(j)}, \quad \xi_{k, 1}^{(j)}=x_{k}^{(j)}+h_{j} / \mu_{k}^{(j)}, \quad \xi_{k, 2}^{(j)}=x_{k+1}^{(j)}-h_{j} / v_{k}^{(j)}, \quad \xi_{k, 3}^{(j)}=x_{k+1}^{(j)} .
$$

Proposition 3. The bidiagonal matrices $S_{k, 1}^{(j)}, S_{k, 2}^{(j)}, S_{k, 3}^{(j)}, S_{k, 4}^{(j)}$ are positive and normalized, i.e. the elements of any row sum up to one, and so both $S_{k}^{(p)}$ and their product $S^{(j)} S^{(j-1)} \ldots S^{(1)} S^{(0)}$ are normalized and totally positive.

In Costantini and Manni (2010a) it is shown that (26) coincides with the well-known de Casteljau algorithm when $\mu_{k}^{(j)}=v_{k}^{(j)}=3$ and, since the matrices $S_{k, 1}^{(j)}, S_{k, 2}^{(j)}, S_{k, 3}^{(j)}$ are bidiagonal, they define a corner cutting process completely similar, even in the rational case $\mu_{k}^{(j)}, v_{k}^{(j)} \neq 3$, to the de Casteljau one.

Proposition 2 says that the points of the sequence $\xi^{(j)}$ are in the correct position stated by $(3)$ in the interval $[0,1]$.

An important consequence of Proposition 3 is that the variation diminishing properties holds for any net $\mathcal{L}_{R}^{(j)}$ with respect to the initial control polygon $\mathcal{L}_{R}^{(0)}$; this, coupled with the total positivity of the Bernstein basis of the spaces (20), ensures us that the functions $\Psi^{(j)}$ are shape preserving, i.e. reproduce the positivity and/or monotonicity and/or convexity of $\mathcal{L}_{R}^{(0)}$.

In order to better understand the modifications of the next section, the subdivision step of (26) is described in Fig. 5. Since in the intervals $\left[x_{k}^{(j)}, x_{k+1}^{(j)}\right], k=1, \ldots, 2^{j}-2$, the subdivision reduces to the well-known de Casteljau algorithm, Fig. 5 reports only one of the significant intervals, namely $k=2^{j}-1$. In the top left part we see the original net at level $j$

$$
\left[\begin{array}{llll}
\xi_{2^{j}-1,0}^{(j)} & \xi_{2^{j}-1,1}^{(j)} & \xi_{2^{j}-1,2}^{(j)} & \xi_{2^{j}-1,3}^{(j)} \\
b_{2^{j}-1,0}^{(j)} & b_{2^{j}-1,1}^{(j)} & b_{2^{j}-1,2}^{(j)} & b_{2^{j}-1,3}^{(j)}
\end{array}\right]^{T}
$$

and, from left to right, the effect of its left multiplication with $S_{2^{j}-1,1}^{(j)}, S_{2^{j}-1,2}^{(j)} S_{2^{j}-1,1}^{(j)}, S_{2^{j}-1,3^{2}}^{(j)} S_{2^{j}-1,2^{2}}^{(j)} S_{2^{j}-1,1}^{(j)}$.

We define the set of extended rational functions

$$
E \mathcal{R}^{(j)}:=\left\{\Psi^{(j)} \text { given in }(23)\right\}
$$

and note that, for any $\Psi^{(j)} \in E \mathcal{R}^{(j)}$ we have

$$
\Psi^{(j)}=\mathcal{B}_{R}^{(j)} S^{(j)} S^{(j-1)} \cdots S^{(1)} S^{(0)} \boldsymbol{b}^{(0)} .
$$

From the linearity of the scheme, $E \mathcal{R}^{(j)}$ is a linear space. More precisely it is a four-dimensional subspace of the $\left(2^{j}+3\right)$ dimensional space of functions of class $C^{2}[0,1]=C^{2}\left[\xi_{0,0}^{(0)}, \xi_{0,3}^{(0)}\right]=C^{2}\left[x_{0}^{(j)}, x_{2^{j}}^{(j)}\right]$ with sections in $\mathcal{R}_{k}^{(j)}, k=0, \ldots, 2^{j}-1$. From Proposition 3 we know that the control polygon $\mathcal{L}_{R}^{(j)}$ lies in the convex hull of $\mathcal{L}_{R}^{(0)}$ and, because of the properties of the Bernstein operator $\mathcal{B}_{R}^{(j)}$, the same holds for $\Psi^{(j)}$ defined by (25). Since the starting functions $\psi^{(0)} \in E \mathcal{R}^{(0)}$ possess tension properties, it follows that, for any $j, E \mathcal{R}^{(j)}$ is a four-dimensional space with tension parameters: increasing the values $\mu_{0}^{(0)}, v_{0}^{(0)}$ any element of $E \mathcal{R}^{(j)}$ approaches the segment through $\left(\xi_{0,0}^{(0)}, b_{0,0}^{(0)}\right),\left(\xi_{0,3}^{(0)}, b_{0,3}^{(0)}\right)$. We remark that in general $E \mathcal{R}^{(j)} \not \equiv E \mathcal{R}^{(0)}=R_{0}^{(0)}$; the only exception is when $\mu_{0}^{(0)}=v_{0}^{(0)}=3$ and in this case we have $E \mathcal{R}^{(j)} \equiv E \mathcal{R}^{(0)} \equiv \mathbb{P}_{3}$.

Let $e_{q}, q=1, \ldots, 4$, denote the elements of the canonical basis of $\mathbb{R}^{4}$. It is possible to construct a basis using the following scheme

$$
B_{R, p}^{(j)}:=\mathcal{B}_{R}^{(j)} S^{(j)} S^{(j-1)} \ldots S^{(1)} S^{(0)} e_{p}^{T}, \quad p=1, \ldots, 4
$$

For $j=0$ the above functions coincide with those specified in Definition 1 for $\mu=\mu_{0}^{(0)}, v=v_{0}^{(0)}$; since the control nets of the basis at level $j$ are obtained via a corner cutting process, the functions of (30) also satisfy (2). Moreover, in Costantini 

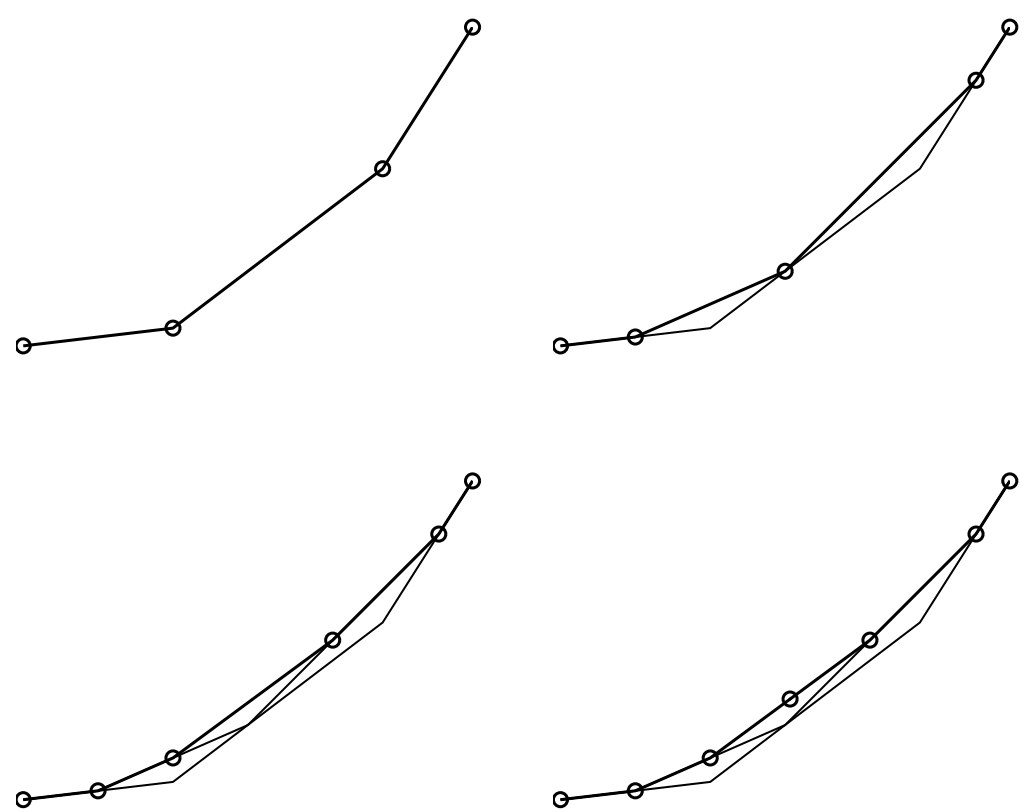

Fig. 5. Graphical representation of the subdivision scheme in the last subinterval $\left[x_{2^{j}-1}^{(j)}, x_{2^{j}}^{(j)}\right]$ with $v_{2^{j}-1}^{(j)}=5$.

and Manni (2010b), Proposition 8, it is proven that the functions $B_{R, 0}^{(j)}, B_{R, 3}^{(j)}$ satisfy condition (4), hence the basis (30) is totally positive and can be referred to as a Bernstein basis. If we set

$$
u_{R}^{(j)}:=B_{R, 0}^{(j)}, \quad v_{R}^{(j)}:=B_{R, 3}^{(j)},
$$

we observe that $E \mathcal{R}^{(j)}$ has the form (1), that is $E \mathcal{R}^{(j)}=\left\langle 1, x, u_{R}^{(j)}, v_{R}^{(j)}\right\rangle$.

\subsection{The space of cubic functions $E \mathcal{C}^{(j)}$}

In the previous subsection we have seen that it is possible to apply an Hermite subdivision scheme to a rational function and obtain at level $j$ a piecewise function with the same properties of cubic polynomials and composed by cubic pieces except in the first and last subinterval. Let us consider (31): aim of this subsection is to substitute the two rational pieces $u_{R}^{(j)}, v_{R}^{(j)}$ with cubic ones $\tilde{u}_{C}^{(j)}, \tilde{v}_{C}^{(j)}$, obtaining the space $E \mathcal{C}^{(j)}$ of $C^{2}$ piecewise cubic functions with tension properties. We first describe the process applying a matrix transformation to a general rational net and then we explain the underlying geometric ideas detailing the construction of $\tilde{v}_{C}^{(j)}$.

Let $\boldsymbol{\xi}^{(j)}, \boldsymbol{b}^{(j)}$, given by (27), define the control net of a generic function $\psi \in E \mathcal{R}^{(j)}$. We define $\tilde{\boldsymbol{\xi}}^{(j)}$, $\tilde{\boldsymbol{b}}^{(j)}$ as

$$
\left[\tilde{\boldsymbol{\xi}}^{(j)}, \tilde{\boldsymbol{b}}^{(j)}\right]=\Sigma^{(j)}\left[\boldsymbol{\xi}^{(j)}, \boldsymbol{b}^{(j)}\right]
$$

where $\Sigma^{(j)} \in \mathbb{R}^{82^{j} \times 82^{j}}$ has the form

$$
\Sigma^{(j)}=\left[\begin{array}{ccccccc}
1 & 0 & 0 & \cdots & 0 & 0 & 0 \\
0 & V\left(\mu_{0}^{(j)}\right) & 1-V\left(\mu_{0}^{(j)}\right) & \cdots & 0 & 0 & 0 \\
0 & 0 & 1 & \cdots & 0 & 0 & 0 \\
\vdots & \vdots & \vdots & \ddots & \vdots & \vdots & \vdots \\
0 & 0 & 0 & \cdots & 1 & 0 & 0 \\
0 & 0 & 0 & \cdots & 1-V\left(v_{2^{j}-1}^{(j)}\right) & V\left(v_{2^{j}-1}^{(j)}\right) & 0 \\
0 & 0 & 0 & \cdots & 0 & 0 & 1
\end{array}\right]
$$

and

$$
V(x)=\frac{x}{2 x-3}
$$


Note that $V^{\prime}(x)<0, V(3)=1, \lim _{x \rightarrow \infty} V(x)=1 / 2$ and so $\Sigma^{(j)}$ defines a corner cutting matrix. Let us recall Proposition 2; since $\mu_{k}^{(j)}=v_{k}^{(j)}=3$ for $k=1, \ldots, 2^{j}-1$, the points $\xi_{k, q}^{(j)}$ coincide with the control points of cubic polynomials, with the exception of the second and second last control points: $\xi_{0,1}^{(j)}, \xi_{2^{j}-1,2}^{(j)}$. Clearly (32) implies that

$$
\tilde{\xi}_{k, q}^{(j)}=\xi_{k, q}^{(j)}, \quad(k, q) \neq(0,1),\left(2^{j}-1,2\right)
$$

and

$$
\tilde{\xi}_{0,1}^{(j)}=\xi_{0,0}^{(j)}+\frac{h_{j}}{3}=\frac{h_{j}}{3}, \quad \tilde{\xi}_{2^{j}-1,2}^{(j)}=\xi_{2^{j}-1,3}^{(j)}-\frac{h_{j}}{3}=1-\frac{h_{j}}{3},
$$

with $h_{j}=2^{-j}$. Therefore all the components of $\tilde{\xi}^{(j)}$ coincide with the abscissas of the control points of cubic polynomials. Let

$$
\tilde{\psi}_{k}^{(j)}(x):=\sum_{q=0}^{3} \tilde{b}_{k, q}^{(j)}\left(\begin{array}{l}
3 \\
q
\end{array}\right)(1-t)^{3-q} t^{q}, \quad t=\frac{x-x_{k}^{(j)}}{h_{j}} ;
$$

note that $\tilde{\psi}_{k}^{(j)}=\psi_{k}^{(j)}, k=1, \ldots, 2^{j}-2$, where $\psi_{k}^{(j)}$ are given in (21). Let

$$
\tilde{\Psi}^{(j)}: \tilde{\Psi}^{(j)}(x)=\tilde{\psi}_{k}^{(j)}(x), \quad x \in\left[x_{k}^{(j)}, x_{k+1}^{(j)}\right],
$$

and let $\mathcal{B}_{C}^{(j)}$ be the linear operator such that $\tilde{\Psi}^{(j)}=\mathcal{B}_{C}^{(j)} \tilde{\boldsymbol{b}}^{(j)}$. We define the space of extended cubic functions

$$
E \mathcal{C}^{(j)}:=\left\{\tilde{\Psi}^{(j)} \text { given in }(33)\right\}
$$

Note that, for any $\tilde{\Psi}^{(j)} \in E \mathcal{C}^{(j)}$,

$$
\tilde{\Psi}^{(j)}=\mathcal{B}_{C}^{(j)} \Sigma^{(j)} S^{(j)} S^{(j-1)} \ldots S^{(0)} \boldsymbol{b}^{(0)} ;
$$

moreover, using the expression for the second derivatives given in (6), it is simple to verify that

$$
D^{2} \tilde{\psi}_{k-1}^{(j)}\left(x_{k}^{(j)}\right)=D^{2} \tilde{\psi}_{k}^{(j)}\left(x_{k}^{(j)}\right)
$$

that is $\tilde{\Psi}^{(j)} \in C^{2}[0,1]$.

It is possible to obtain the analogous of (30):

$$
\tilde{B}_{C, p}^{(j)}:=\mathcal{B}_{C}^{(j)} \Sigma^{(j)} S^{(j)} S^{(j-1)} \cdots S^{(1)} S^{(0)} e_{p}^{T}, \quad p=1, \ldots, 4 .
$$

We recall Definition 1 and state the following property.

Theorem 1. The set $\left\{\tilde{B}_{C, 0}^{(j)}, \tilde{B}_{C, 1}^{(j)}, \tilde{B}_{C, 2}^{(j)}, \tilde{B}_{C, 3}^{(j)}\right\}$ is a Bernstein basis.

Proof. Since the control nets are obtained via a corner cutting process, the functions (36) form a partition of unity and satisfy (2). Using arguments completely similar to those of Proposition 8 of Costantini and Manni (2010b), it is possible to prove that $\tilde{B}_{C, 0}^{(j)}$ and $\tilde{B}_{C, 3}^{(j)}$ satisfy (4) and so the basis (36) is totally positive.

Note that the matrix $\Sigma^{(j)}$ can be decomposed in the product of bidiagonal and stochastic matrices; therefore (32) defines a corner cutting process, the product of the matrices in (35) is a totally positive matrix, since it is a product of totally positive matrices, and $\tilde{\Psi}^{(j)}$ preserves the shape of the original control polygon.

Clearly

$$
E \mathcal{C}^{(j)}=\left\langle\tilde{B}_{C, 0}^{(j)}, \tilde{B}_{C, 1}^{(j)}, \tilde{B}_{C, 2}^{(j)}, \tilde{B}_{C, 3}^{(j)}\right\rangle
$$

and, if we set $\tilde{u}_{C}^{(j)}:=\tilde{B}_{C, 0}^{(j)}, \tilde{v}_{C}^{(j)}:=\tilde{B}_{C, 3}^{(j)}$, then $E \mathcal{C}^{(j)}$ can be written in the form (1), that is

$$
E \mathcal{C}^{(j)}=\left\langle 1, x, \tilde{u}_{C}^{(j)}, \tilde{v}_{C}^{(j)}\right\rangle
$$

Before giving further details a geometric interpretation of the matrix $\Sigma^{(j)}$ is in order. This geometric interpretation also allows the explicit computation of some quantities necessary for practical applications of the space $E \mathcal{C}^{(j)}$. Since the linear process described in (35) is a corner cutting - which leaves unchanged the functions 1 and $x$ - in view of (37) the analysis can be restricted to $\tilde{u}_{C}^{(j)}, \tilde{v}_{C}^{(j)}$ and, in particular, to $\tilde{v}_{C}^{(j)}$ since the other is similar. The basic idea is the following: the four control points of the last rational piece at level $j$, namely

$$
\left(\xi_{2^{j}-1,0}^{(j)}, b_{2^{j}-1,0}^{(j)}\right), \quad\left(\xi_{2^{j}-1,1}^{(j)}, b_{2^{j}-1,1}^{(j)}\right), \quad\left(\xi_{2^{j}-1,2}^{(j)}, b_{2^{j}-1,2}^{(j)}\right), \quad\left(\xi_{2^{j}-1,3}^{(j)}, b_{2^{j}-1,3}^{(j)}\right),
$$




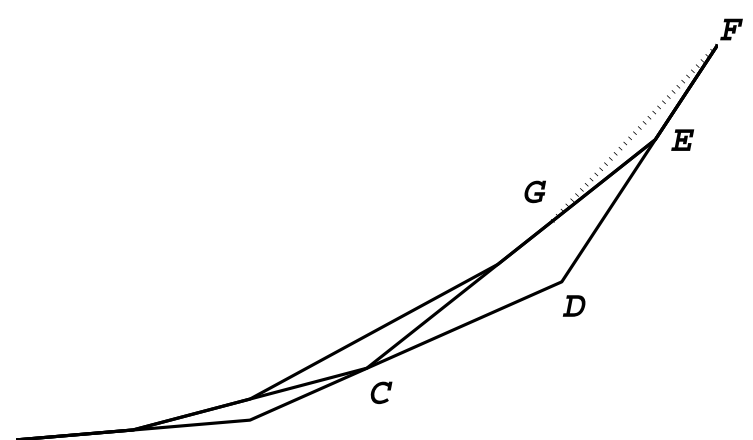

Fig. 6. The modification of the $2^{j}-1$ rational piece.

will be changed into

$$
\left(\tilde{\xi}_{2^{j}-1,0}^{(j)}, \tilde{b}_{2^{j}-1,0}^{(j)}\right), \quad\left(\tilde{\xi}_{2^{j}-1,1}^{(j)}, \tilde{b}_{2^{j}-1,1}^{(j)}\right), \quad\left(\tilde{\xi}_{2^{j}-1,2}^{(j)}, \tilde{b}_{2^{j}-1,2}^{(j)}\right), \quad\left(\tilde{\xi}_{2^{j}-1,3}^{(j)}, \tilde{b}_{2^{j}-1,3}^{(j)}\right),
$$

where

$$
\left(\tilde{\xi}_{2^{j}-1, p}^{(j)}, \tilde{b}_{2^{j}-1, p}^{(j)}\right)=\left(\xi_{2^{j}-1, p}^{(j)}, b_{2^{j}-1, p}^{(j)}\right), \quad p=0,1,3
$$

and

$$
\left(\tilde{\xi}_{2^{j}-1,2}^{(j)}, \tilde{b}_{2^{j}-1,2}^{(j)}\right)=\left(G_{x}, G_{y}\right) .
$$

The scheme is essentially contained in Fig. 6, which shows the subdivision process from level $j-1$ to level $j$ for the last piece in the interval $\left[x_{2^{j-1}-1}^{(j-1)}, x_{2^{j-1}}^{(j-1)}\right]$ and should be compared with Fig. 5. For the sake of readability some of the control points have been relabelled and, in particular,

$$
\begin{aligned}
& \boldsymbol{F}=\left(F_{x}, F_{y}\right)=\left(\xi_{2^{j-1}-1,3}^{(j-1)}, b_{2^{j-1}-1,3}^{(j-1)}\right)=\left(\xi_{2^{j}-1,3}^{(j)}, b_{2^{j}-1,3}^{(j)}\right)=(1,1), \\
& \boldsymbol{D}=\left(D_{x}, D_{y}\right)=\left(\xi_{2^{j-1}-1,2}^{(j-1)}, b_{2^{j-1}-1,2}^{(j-1)}\right), \\
& \boldsymbol{E}=\left(E_{x}, E_{y}\right)=\left(\xi_{2^{j}-1,2}^{(j)}, b_{2^{j}-1,2}^{(j)}\right),
\end{aligned}
$$

$\boldsymbol{C}=\left(C_{x}, C_{y}\right)$ is given by the application of the matrix $S_{2^{j}-1,1}^{(j)}$ (see Fig. 5, top-right) and finally $\boldsymbol{G}=\left(G_{x}, G_{y}\right)$ is the sought for control point of the cubic piece. We recall that

$$
\boldsymbol{D}=\left(1-\frac{1}{2^{j-1} v_{2^{j-1}-1}^{(j-1)}}, 1-\frac{v_{0}^{(0)}}{2^{j-1} v_{2^{j-1}-1}^{(j-1)}}\right), \quad \boldsymbol{E}=\left(1-\frac{1}{\left.2^{j} v_{2^{j}-1}^{(j)}\right)}, 1-\frac{v_{0}^{(0)}}{\left.2^{j} v_{2^{j}-1}^{(j)}\right)}\right) .
$$

Since $G_{x}$ is the abscissa of the control point of a cubic polynomial $G_{x}=1-1 /\left(2^{j} 3\right)$; moreover, it is simple to verify that $C_{x}=\left(x_{2^{j-1}-1}^{(j-1)}+x_{2^{j-1}}^{(j-1)}\right) / 2$. Let

$$
\Delta_{2^{j-1}-1}^{(j-1)}:=\frac{D_{y}-C_{y}}{D_{x}-C_{x}} ; \quad \Delta_{2^{j}-1}^{(j)}:=\frac{E_{y}-C_{y}}{E_{x}-C_{x}}
$$

and

$$
r_{2^{j-1}-1}^{(j-1)}(x):=\Delta_{2^{j-1}-1}^{(j-1)}\left(x-C_{x}\right)+C_{y} ; \quad r_{2^{j}-1}^{(j)}(x):=\Delta_{2^{j}-1}^{(j)}\left(x-C_{x}\right)+C_{y} ;
$$

if we recall (5) we see that $r_{2^{j-1}-1}^{(j-1)}$ and $r_{2^{j}-1}^{(j)}$ are, respectively, the straight lines through the second and third control points of $\psi_{2^{j-1}-1}^{(j-1)}$ and $\psi_{2^{j}-1}^{(j)}$. We have the following result.

Lemma 1. Let $\Delta_{2^{j}-1}^{(j)}$ be given by (38); then

$$
\Delta_{2^{j}-1}^{(j)}=6 \frac{\frac{1}{3}\left(4^{j}-1\right) v_{0}^{(0)}-2^{j}+1}{\left(v_{0}^{(0)}+2^{j+1}-3\right)\left(v_{0}^{(0)}+2^{j}-3\right)} .
$$


Proof. We start by observing that for $v_{R}^{(0)}=B_{R, 3}^{(0)}$ the representation (5) gives $r_{0}^{(0)} \equiv 0$ and thus $\Delta_{0}^{(0)}=0$. It is immediate to see that (40) vanish for $j=0$ and so the claim holds for $j=0$. From (38) and (39) it is not difficult to obtain

$$
\Delta_{2^{\eta+1}-1}^{(\eta+1)}=\frac{\Delta_{2^{\eta}-1}^{(\eta)}\left(\left(v_{2^{\eta}-1}^{(\eta)}\right)^{2}+v_{2^{\eta}-1}^{(\eta)}-6\right)+6 v_{0}^{(0)}}{v_{2^{\eta}-1}^{(\eta)}\left(v_{2^{\eta}-1}^{(\eta)}+1\right)}
$$

If we substitute in (41) the expression of $\Delta_{2^{\eta}-1}^{(\eta)}$ given in (40) and take into account that

$$
v_{2^{\eta}-1}^{(\eta)}=\frac{v_{0}^{(0)}+3\left(2^{\eta}-1\right)}{2^{\eta}}
$$

we obtain

$$
\Delta_{2^{\eta+1}-1}^{(\eta+1)}=6 \frac{\frac{1}{3}\left(4^{\eta+1}-1\right) v_{0}^{(0)}-2^{\eta+1}+1}{\left(v_{0}^{(0)}+2^{\eta+2}-3\right)\left(v_{0}^{(0)}+2^{\eta+1}-3\right)}
$$

and the claim holds applying the induction principle.

For a practical application of the space $E \mathcal{C}^{(j)}$ it is necessary to have an explicit expression of $D \tilde{v}_{C}^{(j)}\left(x_{2^{j}}^{(j)}\right)=D \tilde{v}_{C}^{(j)}(1)$ as a function of $v_{0}^{(0)}=D \Psi_{0}^{(0)}(1)=D \Psi_{2^{j}-1}^{(j)}(1)$. We recall that the restriction of $\tilde{v}_{C}^{(j)}$ to the last dyadic interval $\left[x_{2^{j}-1}^{(j)}, x_{2^{j}}^{(j)}\right]$ is a cubic Bernstein polynomial and so

$$
\beta_{2^{j}-1}^{(j)}:=D \tilde{v}_{C}^{(j)}(1)=\frac{\tilde{b}_{2^{j}-1,3}^{(j)}-\tilde{b}_{2^{j}-1,2}^{(j)}}{\tilde{\xi}_{2^{j}-1,3}^{(j)}-\tilde{\xi}_{2^{j}-1,2}^{(j)}}=\frac{F_{y}-G_{y}}{F_{x}-G_{x}}=\frac{1-G_{y}}{1-G_{x}} .
$$

We have the following result.

Proposition 4. Let $\beta_{2^{j}-1}^{(j)}$ be given by (42); then

$$
\beta_{2^{j}-1}^{(j)}=\beta_{2^{j}-1}^{(j)}\left(v_{0}^{(0)}\right)= \begin{cases}3 & \text { if } j=0, \\ 6 \frac{2^{j-1}\left(v_{0}^{(0)}\right)^{2}+X v_{0}^{(0)}+1}{\left(v_{0}^{(0)}+4 \cdot 2^{j-1}-3\right)\left(v_{0}^{(0)}+2 \cdot 2^{j-1}-3\right)} & \text { if } j>0,\end{cases}
$$

where

$$
X:=\frac{1}{3} \cdot \frac{3+23 \cdot 2^{j-1}-47 \cdot 4^{j-1}-4 \cdot 16^{j-1}+25 \cdot 8^{j-1}}{-4^{j-1}+4 \cdot 2^{j-1}-3} .
$$

Proof. Using (42), the results of Lemma 1 and a computer algebra system we can compute

$$
\beta_{2^{j}-1}^{(j)}=\frac{1-r_{2^{j}-1}^{(j)}\left(1-\frac{1}{3} \frac{1}{2^{j}}\right)}{\frac{1}{3} \frac{1}{2^{j}}}
$$

which can be transformed in the form (43).

Let us consider (42) and Fig. 6; we have

$$
\beta_{2^{j}-1}^{(j)} \leqslant \frac{F_{y}-E_{y}}{F_{x}-E_{x}}=D v_{R}^{(j)}(1)=D v_{R}^{(0)}(1)=v_{0}^{(0)}
$$

We have also the following expression for $\tilde{b}_{2^{j}-1,2}^{(j)}=G_{y}$, directly obtained from the formula $G_{y}=1-\beta_{2^{j}-1}^{(j)}\left(1-G_{x}\right)$. Note that the expressions given below in (45) coincide with that given in implicit form by (32).

\section{Proposition 5.}

$$
\begin{aligned}
& G_{y}=\frac{1}{3} \cdot \frac{a v_{0}^{(0)}+b}{\left(v_{0}^{(0)}+2^{j}-3\right)\left(4 \cdot 2^{j-1}+v_{0}^{(0)}-3\right)\left(-4^{j-1}+4 \cdot 2^{j-1}-3\right)}, \\
& a=31-14 \cdot 8^{j-1}-79 \cdot 2^{j-1}+65 \cdot 4^{j-1}-3 \cdot 2^{-(j-1)}, \\
& b=-93-315 \cdot 4^{j-1}+9 \cdot 2^{-(j-1)}-24 \cdot 16^{j-1}+273 \cdot 2^{j-1}+150 \cdot 8^{j-1} .
\end{aligned}
$$



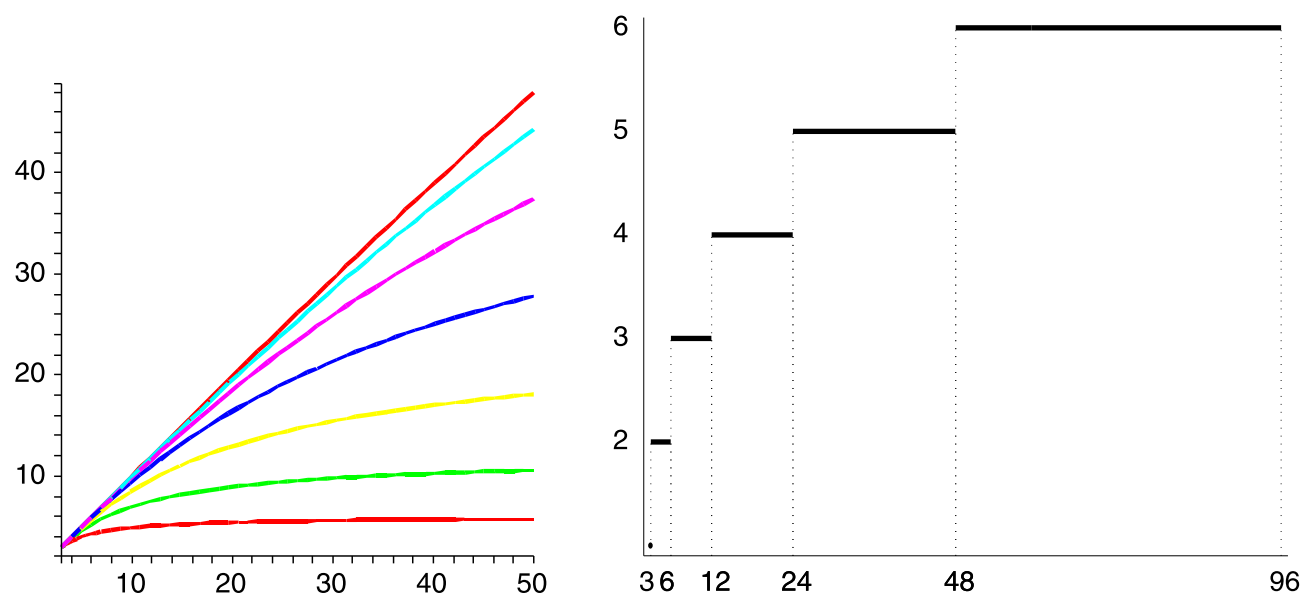

Fig. 7. Left: plots of $\beta_{2^{j}-1}^{(j)}\left(v_{0}^{(0)}\right), j=1, \ldots, 7$. Right: plot of $j(\tilde{\alpha}, \tilde{\alpha})$ for $\tilde{\alpha} \in[3,96]$.

Remark 1. It is clear (see Fig. 6) that the straight lines through the points $\left(\tilde{\xi}_{2^{j}-2,1}^{(j)}, \tilde{b}_{2^{j}-2,1}^{(j)}\right)$, $\left(\tilde{\xi}_{2^{j}-2,2}^{(j)}, \tilde{b}_{2^{j}-2,2}^{(j)}\right)$ and $\left(\tilde{\xi}_{2^{j}-1,1}^{(j)}, \tilde{b}_{2^{j}-1,1}^{(j)}\right),\left(\tilde{\xi}_{2^{j}-1,2}^{(j)}, \tilde{b}_{2^{j}-1,2}^{(j)}\right)$ meet at the point $C$ and so satisfy the $C^{2}$ geometric condition (see Hoschek and Lasser, 1993). This furnishes a geometric proof that $\tilde{v}_{C}^{(j)} \in C^{2}[0,1]$. for

Obviously the arguments above can be repeated for $\tilde{u}_{C}^{(j)}=\tilde{B}_{C, 0}^{(j)}$ and a representation analogous to (43) can be obtained

$$
\alpha_{0}^{(j)}=\alpha_{0}^{(j)}\left(\mu_{0}^{(0)}\right):=D \tilde{u}_{C}^{(j)}(0) .
$$

It follows that it is possible to associate to any $\tilde{\Psi}^{(j)}=\sum_{p=0}^{3} \tilde{c}_{p}^{(j)} \tilde{B}_{C, p}^{(j)}$ a control polygon given by the control points

$$
\left(0, \tilde{c}_{0}^{(j)}\right), \quad\left(\frac{1}{\alpha_{0}^{(j)}}, \tilde{c}_{1}^{(j)}\right), \quad\left(1-\frac{1}{\beta_{2^{j}-1}^{(j)}}, \tilde{c}_{2}^{(j)}\right), \quad\left(0, \tilde{c}_{3}^{(j)}\right) .
$$

The remaining of this subsection is devoted to some practical remarks.

We observe that, in view of (46), the parameters $\alpha_{0}^{(j)}, \beta_{2^{j}-1}^{(j)}$ are tension parameters in the sense specified in Section 2.1.

From formula (35) we see that the construction of $\tilde{\Psi}^{(j)}$ depends on two terms: the starting control net $\boldsymbol{b}^{(0)}$ and the subdivision level $j$. The latter will be discussed later. The shape of $\tilde{\Psi}^{(j)}$ is modelled by the control points (46) which, in turn, depend on the parameters $\alpha_{0}^{(j)}, \beta_{2^{j}-1}^{(j)}$. In view of (43) these parameters, as well as the basis functions (36) depend on $\mu_{0}^{(0)}, v_{0}^{(0)}$. In other words, if we want to construct a function $\tilde{\Psi}^{(j)}$ with suitable values for $\alpha_{0}^{(j)}$, $\beta_{2^{j}-1}^{(j)}$, we must know the corresponding values of $\mu_{0}^{(0)}, v_{0}^{(0)}$ - which define the starting control net $\boldsymbol{b}^{(0)}$ - in order to start the subdivision process described in (35).

From (43) we have immediately that

$$
\beta_{2^{j}-1}^{(j)}(3)=3, \quad \lim _{v_{0}^{(0)} \rightarrow+\infty} \beta_{2^{j}-1}^{(j)}\left(v_{0}^{(0)}\right)=6 \cdot 2^{j-1}, \quad \lim _{j \rightarrow+\infty} \beta_{2^{j}-1}^{(j)}\left(v_{0}^{(0)}\right)=v_{0}^{(0)} .
$$

Fig. 7 (left) shows the behavior of the functions $\beta_{2^{j}-1}^{(j)}\left(v_{0}^{(0)}\right)$. We observe that the denominator of $\beta_{2^{j}-1}^{(j)}$ in (43) is positive for any $j$ when $v_{0}^{(0)} \geqslant 3$; therefore the equation in the unknown $v_{0}^{(0)}$ :

$$
\beta_{2^{j}-1}^{(j)}\left(v_{0}^{(0)}\right)=\tilde{\beta}
$$

can be rewritten as a quadratic equation. On the other hand, we know from (44) and (47) that for any

$$
j>\log _{2}\left(\frac{\tilde{\beta}}{6}\right)+1
$$

one of the two real roots of (48) lyes in $\left[3, v_{0}^{(0)}\right]$ (indeed, numerical experiments show that the other root is negative). We have therefore the following result. 
Proposition 6. Let $v 1, v 2$ be the two roots of (48). Then we chose the solution of (48) as

$$
v_{0}^{(0)}:=\max \{v 1, v 2\}
$$

Obviously completely similar consideration can be done for the parameter $\alpha_{0}^{(j)}\left(\mu_{0}^{(0)}\right)$ associated with $x=0$, the equation

$$
\alpha_{0}^{(j)}\left(\mu_{0}^{(0)}\right)=\tilde{\alpha}
$$

has a structure identical to (48) and the correct root $\alpha_{0}^{(0)}$ can be chosen according to the rule stated in Proposition 6 . Finally, it remains to fix the choice for the integer $j=j(\tilde{\alpha}, \tilde{\beta})$; taking into account (49) and the graphical results of many practical experiments we use the following formula:

$$
j=1+\max \left\{\left\lceil\log _{2}\left(\frac{\tilde{\alpha}}{6}\right)+1\right\rceil,\left\lceil\log _{2}\left(\frac{\tilde{\beta}}{6}\right)+1\right\rceil\right\}
$$

where

$$
\lceil x\rceil=\min \{z \in \mathbb{Z} \text { s.t. } x \leqslant z\} .
$$

A plot of $j(\tilde{\alpha}, \tilde{\alpha})$ is shown in Fig. 7 (right). It is possible to see the modest increase of the subdivision level when $\tilde{\alpha}$ increases.

Remark 2. Note that even if for a level $j$ the number of dyadic subintervals is $2^{j}$, (24) assures that the number of cubic pieces, counting in the present case also $\left[x_{0}^{(j)}, x_{1}^{(j)}\right]$ and $\left[x_{2^{j}-1}^{(j)}, x_{2^{j}}^{(j)}\right]$, is $2 j$ for $j \geqslant 1$.

In conclusion, $\tilde{\Psi}^{(j)} \in E \mathcal{C}^{(j)}$ can be constructed with the following steps.

- Compute $\tilde{\alpha}, \tilde{\beta}$ according to some shape criterion.

- Compute $j$ using (50).

- Compute $\mu_{0}^{(0)}, v_{0}^{(0)}$ using the method of Proposition 6.

- Compute $\tilde{\Psi}^{(j)}$ using (35) and taking into account Remark 2.

Note that if $\tilde{\alpha}=\tilde{\beta}=3$ then $\mu_{0}^{(0)}=v_{0}^{(0)}=3$ and so $\tilde{u}_{C}^{(j)}(x)=(1-x)^{3}, \tilde{v}_{C}^{(j)}(x)=x^{3}$, that is $E \mathcal{C}^{(j)}=\mathbb{P}_{3}$. For further references, we will also use the notation

$$
E \mathcal{C}^{(j)}=E \mathcal{C}^{(j)}(\tilde{\alpha}, \tilde{\beta})
$$

which puts in evidence the dependence on the shape parameters.

We conclude this section with an expression for $D^{2} \tilde{u}_{C}^{(j)}(0)$ and $D^{2} \tilde{v}_{C}^{(j)}(1)$. This expression is obtained using formula (6) and a computer algebra system.

Theorem 2. We have

$$
D^{2} \tilde{v}_{C}^{(j)}(1)=D^{2} \tilde{v}_{C}^{(j)}\left(1 ; v_{0}^{(0)}\right)=\frac{24\left(a\left(v_{0}^{(0)}\right)^{4}+b\left(v_{0}^{(0)}\right)^{3}+c\left(v_{0}^{(0)}\right)^{2}+d v_{0}^{(0)}+e\right)}{\left(v_{0}^{(0)}+2^{j}-3\right)\left(v_{0}^{(0)}+2^{j-1}-3\right)\left(4 \cdot 2^{j-1}+v_{0}^{(0)}-3\right)\left(v_{0}^{(0)}+3 \cdot 2^{j-1}-3\right)}
$$

where

$$
\begin{array}{ll}
a=4^{j-2}, \quad b=\left(-9 \cdot 4^{j-2}+4 \cdot 8^{j-2}\right), & c=\left(29 \cdot 4^{j-2}+3 \cdot 16^{j-2}-24 \cdot 8^{j-2}\right), \\
d=\left(-39 \cdot 4^{j-2}+44 \cdot 8^{j-2}-9 \cdot 16^{j-2}\right), & e=-24 \cdot 8^{j-2}+18 \cdot 4^{j-2}+6 \cdot 16^{j-2},
\end{array}
$$

and, since by construction $\tilde{u}_{C}^{(j)}\left(x ; \mu_{0}^{(0)}\right)=\tilde{v}_{C}^{(j)}\left(1-x ; \mu_{0}^{(0)}\right)$,

$$
D^{2} \tilde{u}_{C}^{(j)}(0)=D^{2} \tilde{u}_{C}^{(j)}\left(0 ; \mu_{0}^{(0)}\right)=D^{2} \tilde{v}_{C}^{(j)}\left(1 ; \mu_{0}^{(0)}\right)=D^{2} \tilde{v}_{C}^{(j)}(1)
$$




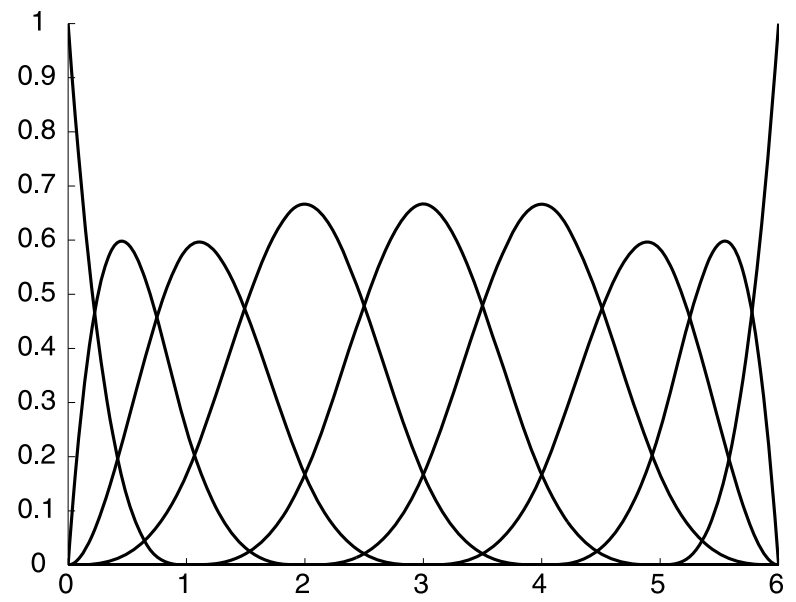

Fig. 8. Plot of a B-spline basis. $m_{0}=m_{6}=4, m_{i}=1 ; \lambda_{i}=3$.

\section{The spline space}

In the previous section we have constructed the space $E \mathcal{C}^{(j)}$ which is a space of generalized cubics, that is has the properties stated in Section 2.1. Our next goal is to apply the results of Section 2.3 and obtain generalized splines, piecewise composed by functions of the form (33). Let us consider the knot sequences (10), (11) and the multiplicities $m_{0}, \ldots, m_{n}$. The distinct knots are equipped with tension parameters

$$
\lambda_{0}, \lambda_{1}, \ldots, \lambda_{n} .
$$

We associate with any subinterval $\left[\bar{y}_{i}, \bar{y}_{i+1}\right]$ a space of the form (34), (37) ${ }^{4}$

$$
E \mathcal{C}_{i}^{\left(j_{i}\right)}\left(\tilde{\alpha}_{i}, \tilde{\beta}_{i}\right)=E \mathcal{C}_{i}^{\left(j_{i}\right)}\left(\lambda_{i}, \lambda_{i+1}\right)=\left\langle 1, t, \tilde{u}_{i, C}^{\left(j_{i}\right)}(t), \tilde{v}_{i, C}^{\left(j_{i}\right)}(t)\right\rangle, \quad t=\frac{x-\bar{y}_{i}}{h_{i}},
$$

and compute any $\tilde{\Psi}_{i}^{\left(j_{i}\right)} \in E \mathcal{C}_{i}^{\left(j_{i}\right)}\left(\lambda_{i}, \lambda_{i+1}\right)$ with the four steps described in Section 3.2. We define a spline space of the form (12) as

$$
\tilde{S}_{C}:=\left\{s \text { s.t. } s_{i}:=s_{\mid\left[\bar{y}_{i}, \bar{y}_{i+1}\right)} \in E \mathcal{C}_{i}^{\left(j_{i}\right)}\left(\lambda_{i}, \lambda_{i+1}\right), i=0, \ldots, n-1 ; s \in C^{3-m_{i}}\left(\bar{y}_{i}\right), i=1, \ldots, n-1\right\} .
$$

The construction of the B-spline basis for $\tilde{S}_{C}$

$$
\tilde{\mathcal{N}}_{0}, \tilde{\mathcal{N}}_{1}, \ldots, \tilde{\mathcal{N}}_{N}
$$

is completely defined in Section 2.3; in particular in (14) we use $\rho_{i}=1 / \lambda_{i}, \omega_{i}=1 / \lambda_{i+1}$ and in (17) the values of $D^{2} \tilde{u}_{i, C}^{\left(j_{i}\right)}(0)$, $D^{2} \tilde{v}_{i, C}^{\left(j_{i}\right)}(1)$ are given in Theorem 2. Some examples of B-spline bases are given in Figs. 8-10. In order to better understand the influence of knot's multiplicities and shape parameters the distinct knots are equidistant. Fig. 8 represents the classical $C^{2}$ cubic B-spline basis and should be used for comparison with the other plots.

\section{Examples and conclusions}

In the previous section we have shown the construction of cubic like B-splines with tension properties. Any conceivable application - interpolation, approximation, free-form design, extension to tensor product and Boolean sum surfaces, etc. can be easily obtained substituting in the standard algorithms the classical cubic B-splines with the functions (51). For limit of space, in Figs. 11-15 we give only some examples in free-form design, using $C^{2}$ planar curves or tensor-product surfaces with simple knots and obtained with different shape parameters. Fig. 11 reports a standard cubic spline curve and should be used for comparison with the other plots. For all the curves we have used chord-length parameterization.

Figs. 14-15 present the control points and the corresponding tensor-product surfaces. In this case we have two sequences of shape parameters, say $\lambda_{0}^{(w)}, \ldots, \lambda_{n_{w}}^{(w)}$ and $\lambda_{0}^{(z)}, \ldots, \lambda_{n_{z}}^{(z)}$, associated respectively to the knots $w_{0}, \ldots, w_{n_{w}}$ and $z_{0}, \ldots, z_{n_{z}}$. We used uniform parameterization.

We conclude the paper with a final remark.

\footnotetext{
${ }^{4}$ It is possible (and simple) to associate two parameters for each knot so that $\tilde{\beta}_{i-1} \neq \tilde{\alpha}_{i}$. However, our experience in practical shape preserving problems suggests that the "one parameter choice" results in the best combination both of shape control and simplicity.
} 

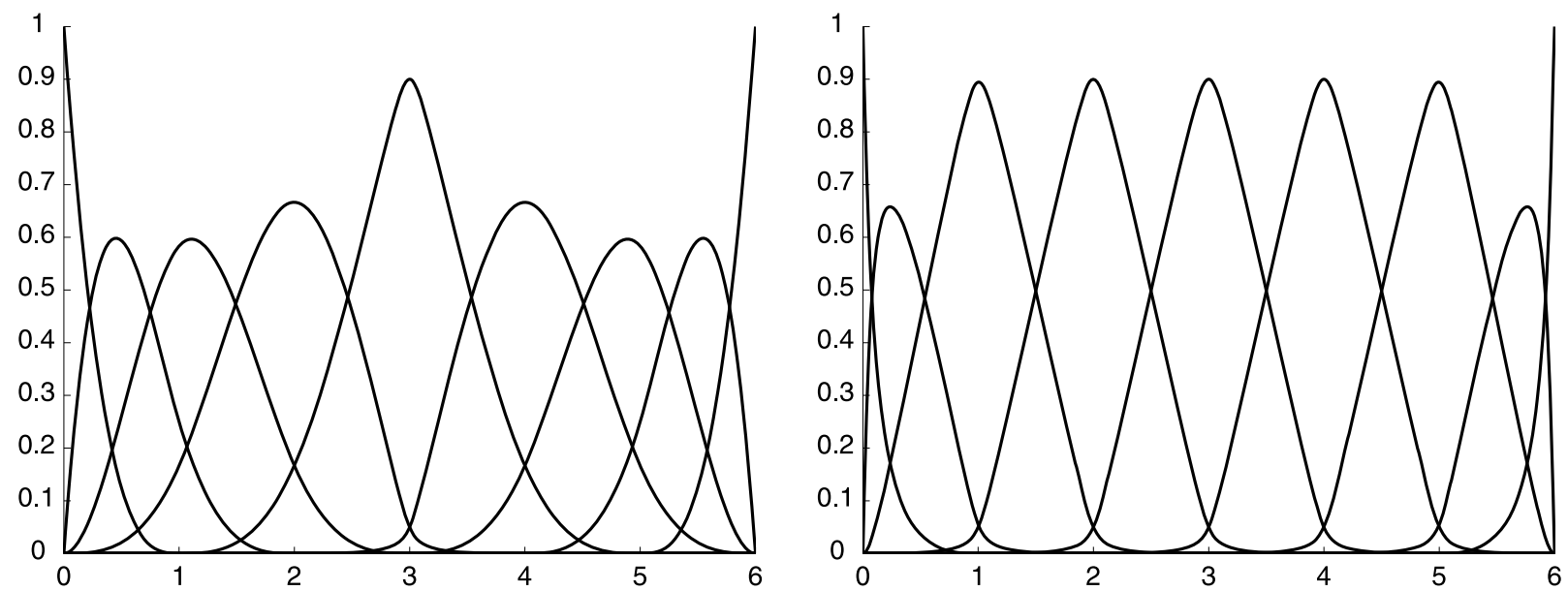

Fig. 9. Plot of a B-spline basis. Left: $m_{0}=m_{6}=4, m_{i}=1 ; \lambda_{3}=10, \lambda_{i}=3$. Right: $m_{0}=m_{6}=4, m_{i}=1 ; \lambda_{i}=10$.
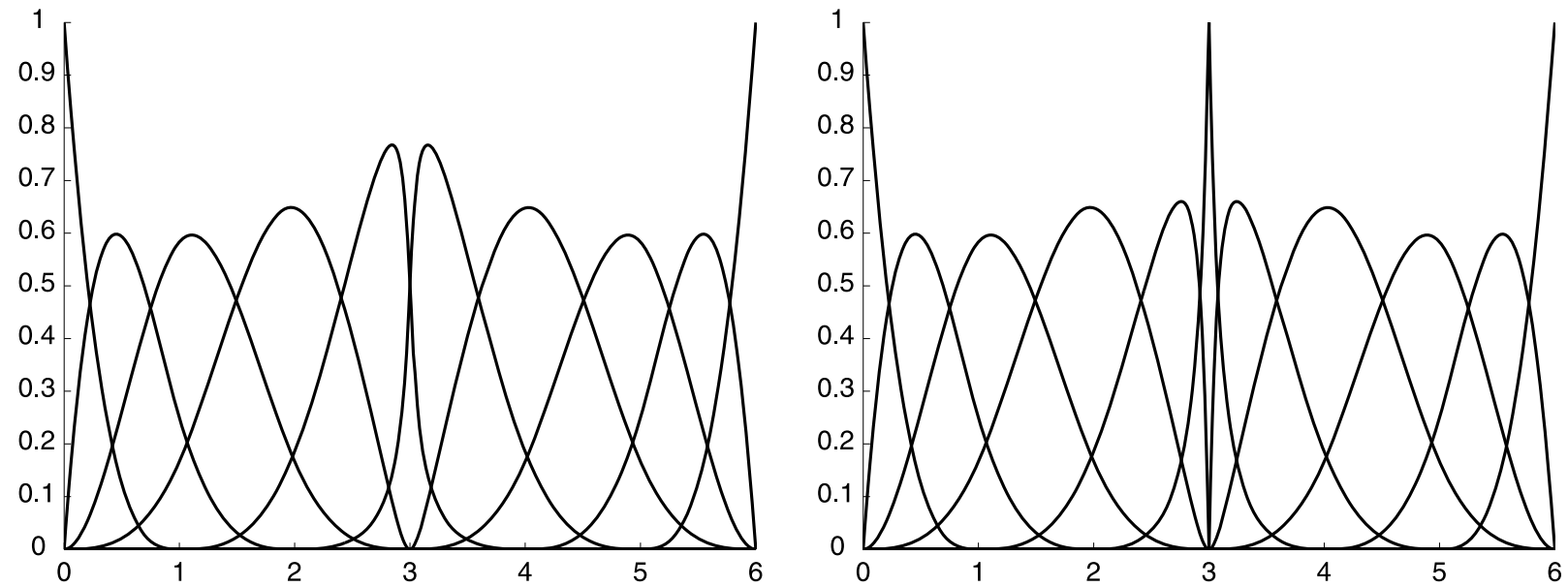

Fig. 10. Plot of a B-spline basis. Left: $m_{0}=m_{6}=4, m_{3}=2 m_{i}=1 ; \lambda_{3}=10, \lambda_{i}=3$. Right: $m_{0}=m_{6}=4, m_{3}=3, m_{i}=1 ; \lambda_{3}=10, \lambda_{i}=3$.

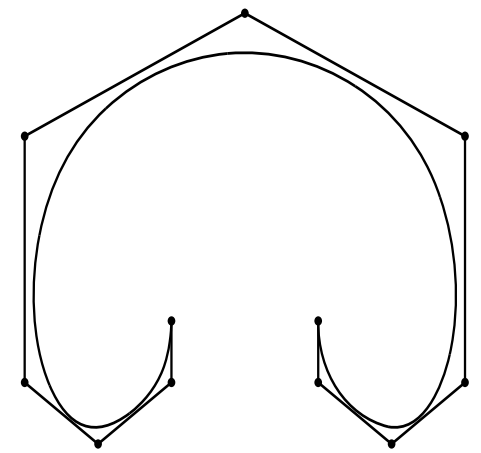

Fig. 11. Plot of an open B-spline curve. $\lambda_{i}=3$.

We have presented a method for the construction of cubic like B-splines with multiple knots, composed by extended cubic polynomials $\tilde{\Psi}_{i} \in E \mathcal{C}_{i}^{(j)}$, where $j$ is related to a subdivision scheme. Such extended cubic polynomials are in turn composed by $2 j$ cubic polynomials pieces which connect with $C^{2}$ continuity. The proposed B-splines are therefore conformal to the NURBS based CAD/CAM standard. In addition, they are equipped with tension parameters, associated to the knots, which permit a modification of their shape. The properties and applications of these splines in shape preserving interpolation or approximation and in free form design are completely similar to the many tension methods appeared in the last two decades. It is worth remarking that such tension methods could not be inserted in standard software because do not give 

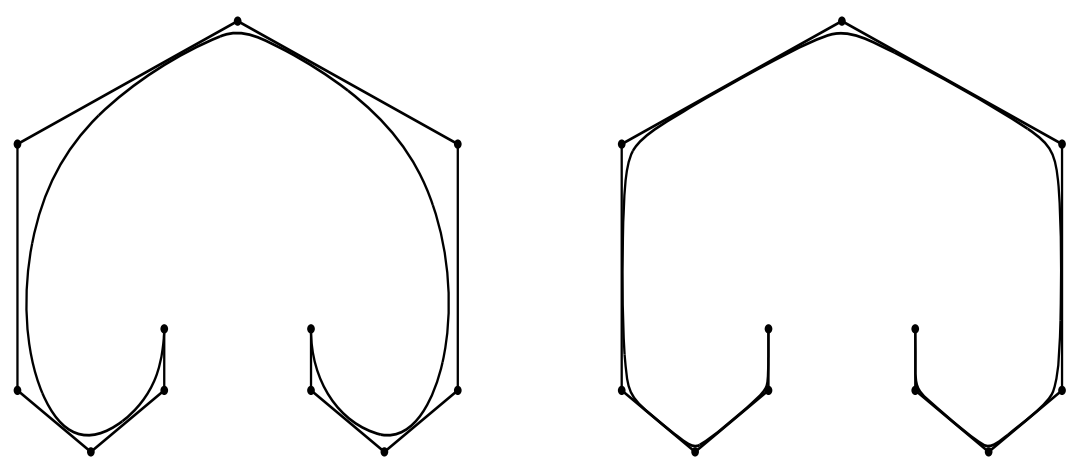

Fig. 12. Plot of an open B-spline curve. Left: $\lambda_{5}=10, \lambda_{i}=3$. Right: $\lambda_{i}=10$.
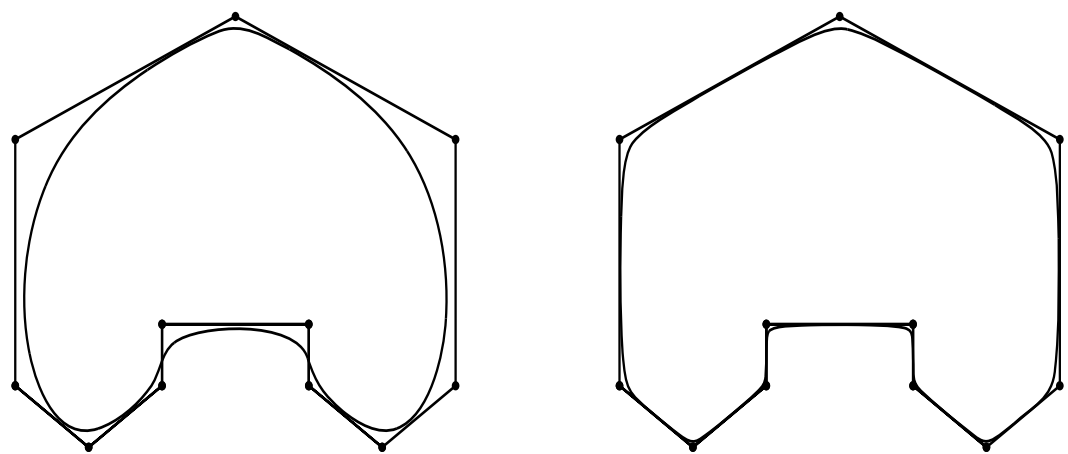

Fig. 13. Plot of a closed B-spline curve. Left: $\lambda_{5}=10, \lambda_{i}=3$. Right: $\lambda_{i}=10$.
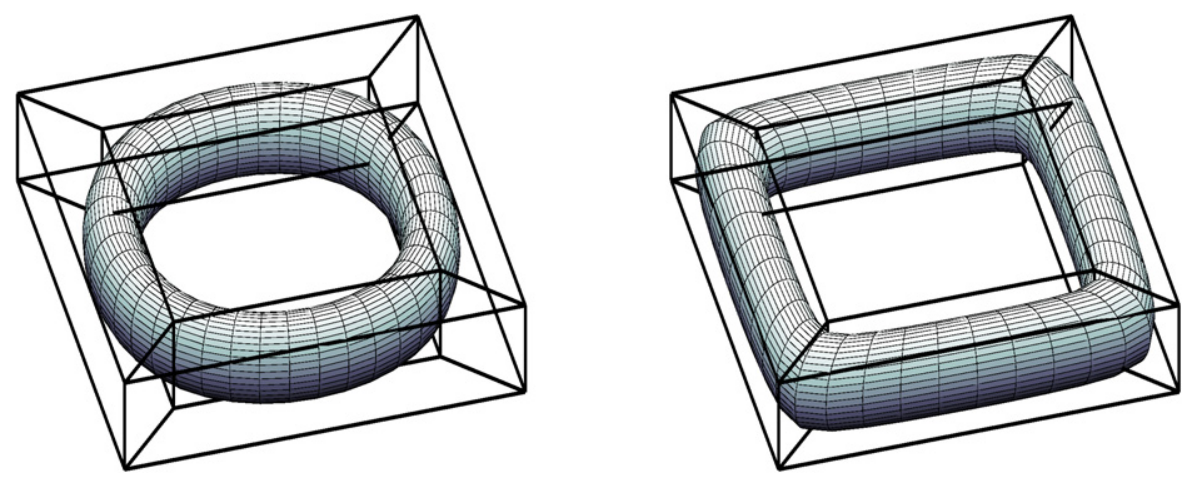

Fig. 14. Control points and the spline surface. Left: $\lambda_{i}^{(w)}=3$ all $i ; \lambda_{j}^{(z)}=3$ all $j$. Right: $\lambda_{i}^{(w)}=10$ all $i ; \lambda_{j}^{(z)}=3$ all $j$.
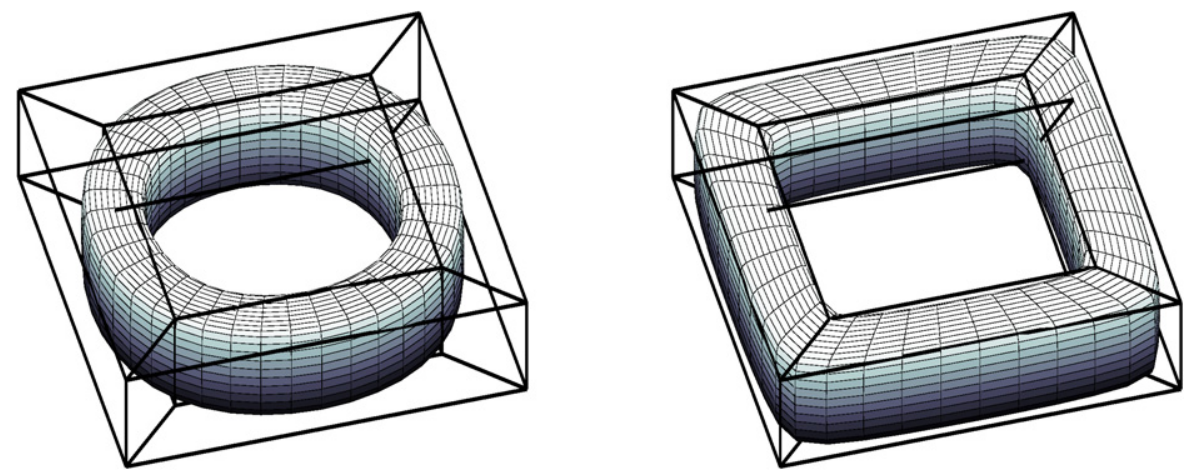

Fig. 15. Control points and the spline surface. Left: $\lambda_{i}^{(w)}=3$ all $i ; \lambda_{j}^{(z)}=10$ all $j$. Right: $\lambda_{i}^{(z)}=10$ all $i ; \lambda_{j}^{(w)}=10$ all $j$. 
explicit B-splines construction and/or are not composed by low degree NURBS; therefore we believe that the proposed techniques could definitely improve the performances of CAD/CAM systems.

\section{Acknowledgements}

We would like to thank the referees for the encouraging reports and the useful comments.

\section{References}

Costantini, P., 2000. Curve and surface construction using variable degree polynomial splines. Comput. Aided Geom. Design 17, $426-446$.

Costantini, P., Manni, C., 2006. Geometric construction of generalized cubic splines. Rend. Mat. 26, 327-338.

Costantini, P., Manni, C., 2010a. A geometric approach for Hermite subdivision. Num. Math. 115, 333-369.

Costantini, P., Manni, C., 2010b. Curve and surface construction using Hermite subdivision schemes. J. Comput. Appl. Math. 233 , $1660-1673$.

Costantini, P., Lyche, T., Manni, C., 2005. On a class of weak Tchebycheff systems. Num. Math. 101, 333-354.

Delbourgo, R., 1989. Shape preserving interpolation to convex data by rational functions with quadratic numerator and linear denominator. IMA J. Numer.

Anal. 1, 123-136.

Delbourgo, R., Gregory, J.A., 1985. Shape preserving piecewise rational interpolation. SIAM J. Sci. Stat. Comput. 6, 967-976

Goodman, T.N.T., Mazure, M.L., 2001. Blossoming beyond extended Chebyshev spaces. J. Approx. Theory 109, $48-81$.

Hoschek, J., Lasser, D., 1993. Fundamentals of Computer Aided Geometric Design. A.K. Peters, Ldt., Wellesley, Massachusetts.

Kaklis, P.D., Pandelis, D.G., 1990. Convexity preserving Polynomial splines of non-uniform degree. IMA J. Numer. Anal. 10, $223-234$

Kaklis, P.D., Sapidis, N.S., 1995. Convexity preserving interpolatory parametric splines of non-uniform polynomial degree. Comput. Aided Geom. Design 12,

$1-26$.

Koch, P.E., Lyche, T., 1993. Interpolation with exponential B-splines in tension. Comput. Suppl. 8, 173-190.

Merrien, J.-L., 1992. A family of Hermite interpolants by bisection algorithms. Num. Alg. 2, 187-200.

Peña, J.M. (Ed.), 1995. Shape Preserving Representations in Computer-Aided Geometric Design. Nova Science Publishers, Inc., Commack, New York.

Sablonnière, P., 2004. Bernstein-type bases and corner cutting algorithms for $C^{1}$ Merrien's curves. Adv. Comp. Math. 20, $229-246$.

Schweikert, D.G., 1966. Interpolatory tension splines with automatic selection of tension factors. J. Math. Phys. 45, $312-327$.

Späth, H., 1969. Exponential spline interpolation. Computing 4, 225-233. 